TRANSACTIONS OF THE

AMERICAN MATHEMATICAL SOCIETY

Volume 358, Number 8, Pages 3329-3361

S 0002-9947(06)04021-9

Article electronically published on February 20, 2006

\title{
KIRWAN-NOVIKOV INEQUALITIES ON A MANIFOLD WITH BOUNDARY
}

\author{
MAXIM BRAVERMAN AND VALENTIN SILANTYEV
}

\begin{abstract}
We extend the Novikov Morse-type inequalities for closed 1-forms in 2 directions. First, we consider manifolds with boundary. Second, we allow a very degenerate structure of the critical set of the form, assuming only that the form is non-degenerated in the sense of Kirwan. In particular, we obtain a generalization of a result of Floer about the usual Morse inequalities on a manifold with boundary. We also obtain an equivariant version of our inequalities.

Our proof is based on an application of the Witten deformation technique. The main novelty here is that we consider the neighborhood of the critical set as a manifold with a cylindrical end. This leads to a considerable simplification of the local analysis. In particular, we obtain a new analytic proof of the Morse-Bott inequalities on a closed manifold.
\end{abstract}

\section{INTRODUCTION}

0.1. The main results. In 7, 10, Michael Farber and the first author extended the Novikov Morse-type inequalities for a closed 1-form $\omega$ on a closed manifold to the case when the critical set of $\omega$ consists of a disjoint union of smooth submanifolds non-degenerate in the sense of Bott. The Novikov inequalities were also considerably strengthened by means of twisting by an arbitrary flat vector bundle $\mathcal{F}$.

In this paper we further extend the results of [7, 10] by considering a manifold $M$ with boundary and by allowing much more degenerate critical sets of $\omega$. For our inequalities to be valid we have to assume that near the boundary the 1-form is exact and can be written as 1

$$
\omega=d\left(f(x) t^{m} / m\right), \quad m>0,
$$

where $x$ is a coordinate on the boundary $\Gamma=\partial M$ of $M, t>0$ is an additional coordinate such that $\Gamma=\{t=1\}$, and $f$ is a smooth function on $\Gamma$ for which zero is a regular value. Set

$$
U^{-}:=\{x \in \Gamma: f(x)<0\} .
$$

If the form $\omega$ is exact, then the role of the Betti numbers in our Morse-type inequalities is played by the dimensions of the relative cohomology $H^{\bullet}\left(M, U^{-} ; \mathcal{F}\right)$ of the

Received by the editors April 9, 2004.

2000 Mathematics Subject Classification. Primary 57R70; Secondary 58A10.

Key words and phrases. Novikov inequalities, Kirwan inequalities, Morse-Bott inequalities, Witten deformation.

This research was partially supported by NSF grant DMS-0204421.

${ }^{1}$ In fact, our results are valid under a slightly weaker assumption. See Section 1 for the exact formulation. 
topological pair $\left(M, U^{-}\right)$with coefficients in a flat vector bundle $\mathcal{F}$. In general, we define the relative Novikov numbers of the topological pair $\left(M, U^{-}\right)$with coefficients in a flat vector bundle $\mathcal{F}$, which enter our inequalities; cf. Definition 1.10. Our construction of the relative Novikov numbers is similar to Pazhitnov's construction of the usual Novikov numbers as generic values of the dimensions of the cohomology of a 1-parameter family of flat connections [25] (see also [10]).

When applied to an exact 1-form $\omega=d h$, our inequalities extend the KirwanMorse inequalities to manifolds with boundary. For the case when the critical points of $\omega$ are isolated and $\mathcal{F}=\mathbb{C}$ is a trivial line bundle, similar inequalities were obtained by Floer 19 .

The condition (0.1) is equivalent to the assumption that $\omega$ can be extended to a homogeneous 1-form on the manifold

$$
\widetilde{M}=M \cup(\Gamma \times[1, \infty)),
$$

which does not have zeros on $\Gamma \times[1, \infty)$; cf. Section 3 . We refer to $\widetilde{M}$ as a manifold with a cylindrical end. Thus we also obtain Morse-type inequalities for homogeneous 1-forms on a manifold with a cylindrical end, which generalize the Morse inequalities for generating functions quadratic at infinity, used in the theory of Lagrangian intersections [29, 16].

We also obtain an equivariant version of our inequalities, which extends the results of [8, 9] to manifolds with boundary and to 1-forms whose critical sets are not manifolds. In particular we obtain equivariant Morse inequalities on a manifold with boundary. It is also important that we consider Morse-type inequalities twisted by a flat vector bundle $\mathcal{F}$. In many examples, this gives much stronger inequalities than the usual Morse-type inequalities with coefficients in $\mathbb{C}$; cf. Example 1.7 of [10. This is even more important for equivariant inequalities; cf. [8] and also the discussion in Section 2

0.2. The method of the proof. Our proof is based on an application of a version of the Witten deformation technique applied to the extension of all the structures to the manifold (0.2). Though a large part of our proof is quite standard, there are two new ideas involved. First, the structure of our Witten-type deformation near the critical set of $\omega$ is new. To explain the novelty, let us temporarily assume that $\omega$ is non-degenerate in the sense of Bott. Recall that the Witten technique is based on considering a one-parameter deformation $\Delta_{T}(T \in \mathbb{R})$ of the Laplacian and comparing the spectrum of $\Delta_{T}$ for large $T$ with the spectrum of a similar operator $\Delta_{T}^{N}$ on the normal bundle to the critical set of $\omega$. It is crucial that the eigenfunctions of both operators $\Delta_{T}$ and $\Delta_{T}^{N}$ concentrate near the critical set for large $T$ for the method to work. It is well known, however, that if the critical points of $\omega$ are not isolated and if we endow the bundle $N$ with a natural "bundle-like" Riemannian metric, then the naive generalization of the Witten construction does not work, because the eigenfunctions of $\Delta_{T}^{N}$ do not concentrate to the critical set as $T \rightarrow \infty$; cf. [5]. This problem was solved by Bismut [5], who proposed a smart two-parameter family of deformations of the Laplacian (the Bismut deformation). It is not clear, however, how to define this two-parameter family when the critical set of $\omega$ is not a manifold.

In this paper we apply a different idea. We consider the bundle $N$ as a manifold with a cylindrical end and introduce a Riemannian metric on $N$ which is conical on 
the cylindrical end; cf. Sections 4 and 6 One of the advantages of this approach is that now the analysis of the spectrum of the operator $\Delta_{T}^{N}$ is exactly the same as the analysis of the spectrum of the operator $\Delta_{T}^{\widetilde{M}}$ on the manifold $\widetilde{M}$. In particular, the eigenfunctions of $\Delta_{T}^{N}$ concentrate near the critical set and the kernel of $\Delta_{T}^{N}$ can be calculated in cohomological terms; cf. Sections 4 and Section 5 .

The second new element of our proof is the study of the Witten Laplacian on a manifold with a cylindrical end (which we need for the study of both operators $\Delta_{T}^{N}$ and $\Delta_{T}^{\widetilde{M}}$ ). In Section 5 , we calculate the dimension of the kernels of the Witten Laplacians on a manifold with a cylindrical end and show that they are equal to the relative Novikov numbers of the topological pair $\left(M, U^{-}\right)$with coefficients in a flat vector bundle $\mathcal{F}$. This is probably the most non-trivial part of our paper.

0.3. The structure of the paper. In Section 1 we formulate our main result and discuss some of its applications and implications.

In Section 2 we present an equivariant version of our inequalities.

In Section 3 we reformulate our main theorem in terms of a manifold with a cylindrical end and show the equivalence of the two formulations.

In Section 4 we define the Witten-type deformation of the Laplacian on a manifold with a cylindrical end and show that it has a discrete spectrum.

In Section 5 we show that the dimension of the kernel of the Witten Laplacian is given by the relative Novikov numbers.

In Section [ 6 we introduce a structure of a manifold with a cylindrical end on a neighborhood of the critical set of $\omega$ and describe the structure of our Witten-type deformation near the critical set.

In Section 7 we present a proof of the Kirwan-Novikov inequalities, based on a result of a comparison between the spectrum of the Witten Laplacians on $\widetilde{M}$ and on $N$. This result is proven in Section 8 .

\section{Preliminaries AND The MAin Result}

1.1. Let $M$ be a compact manifold with boundary $\Gamma=\partial M$. Note that we do not exclude the case when $\Gamma$ is empty. If the boundary is not empty we will identify its tubular neighborhood $\mathcal{U}$ with the product $\Gamma \times(0,1]$ and we will identify points of $\mathcal{U}$ with pairs $(x, t), x \in \Gamma, t \in(0,1]$.

Let $\omega$ be a closed 1-form on $M$ such that the restriction of $\omega$ to $\mathcal{U}$ is exact. In other words we assume that there exists a smooth function $h: \Gamma \times(0,1] \rightarrow \mathbb{R}$ such that

$$
\omega(x, t)=d h(x, t)=d_{\Gamma} h(x, t)+\frac{\partial h}{\partial t}(x, t) d t, \quad x \in \Gamma, t \in(0,1] .
$$

Here $d_{\Gamma}$ is the de Rham differential on $\Gamma$.

1.2. Assumptions on $\omega$ at the boundary. We assume that

(B1) $d_{\Gamma} h(x, 1)+\frac{\partial h}{\partial t}(x, 1) d t \neq 0$ for all $x \in \Gamma$. This implies that $\omega$ does not have zeros at a neighborhood of $\partial M$. Without loss of generality we can and we will assume that $\omega$ does not have zeros at $\Gamma \times(0,1]$.

(B2) 0 is a regular value of the function $\frac{\partial h}{\partial t}(x, 1)$.

(B3) If $\frac{\partial h}{\partial t}(x, 1)=0$, then

$$
d_{\Gamma} h(x, 1) \neq-\lambda \cdot d_{\Gamma}\left(\frac{\partial h}{\partial t}(x, 1)\right), \quad \text { for all } \quad \lambda>0 .
$$


The assumptions (B1) and (B2) mean that $\omega$ is in a generic position near $\partial M$. They can be always achieved by a small perturbation of $h$. Note also that (B2) implies, in particular, that the open set

$$
U^{-}=\left\{x \in \Gamma: \frac{\partial h}{\partial t}(x, 1)<0\right\} \times\{1\} \subset \partial M
$$

has a smooth boundary.

The assumption (B3) is more restrictive. It is essentially equivalent to the fact that the closure $\overline{U^{-}}$of $U^{-}$is the exit set in the sense of Conley [13, 19, for the flow of the vector field $-\nabla h$. In Section 3 we also explain that this condition is necessary to extend $\omega$ to a "homogeneous at infinity" form on $M \cup(\Gamma \times(1, \infty))$. Also in Subsection 1.15 we give an example which shows that without this assumption our main theorem (Theorem 1.12) is not valid.

1.3. Example. Suppose that on near $\Gamma$ we have $h(x, t)=\frac{1}{m} f(x) t^{m}, m>0$. Then condition (B3) is automatically satisfied, while conditions (B1) and (B2) hold if and only if 0 is a regular value of $f(x)$. This example is the most important for applications. In fact, in Section 3 we will show that if the conditions (B1)-(B3) are satisfied, then one can always extend $\omega$ and $h$ to a bigger manifold $M^{\prime}=$ $M \cup(\Gamma \times[1, A])$ without producing new zeros so that near the boundary of $M^{\prime}$ we have $h(x, t)=f(x) t^{2} / 2$.

1.4. Non-degeneracy assumptions on the zeros of $\omega$. We assume that the set

$$
\mathbf{C}=\{x \in M: \omega(x)=0\},
$$

called the critical set of $\omega$, belongs to $M \backslash \mathcal{U}$ and satisfies the following non-degeneracy condition of Kirwan [22]:

Definition 1.5. A closed 1-form $\omega$ is called minimally degenerate or non-degenerate in the sense of Kirwan if it satisfies the following conditions:

(C1) The critical set $\mathbf{C}$ is a finite union of disjoint closed subsets $C \subset \mathbf{C}$ called critical subsets of $\omega$. In a neighborhood of each subset $C$ there exists a smooth function $h_{C}$ such that $\omega=d h_{C}$ and $h_{C}(x)=0$ for all $x \in C$.

(C2) For every $C \subset \mathbf{C}$ there exists a locally closed connected submanifold $\Sigma_{C}$, called a minimizing manifold, containing $C$ such that $\left.h_{C}\right|_{\Sigma_{C}} \geq 0$ and

$$
C=\left\{x \in \Sigma_{C}: h_{C}(x)=0\right\} .
$$

In other words, $C$ is the subset of $\Sigma_{C}$ on which $h_{C}$ takes its minimum values.

(C3) At every point $x \in C$ the tangent space $T_{x} \Sigma_{C}$ is the maximal among all subspaces of $T_{x} M$ on which the Hessian $H_{x}\left(h_{C}\right)$ is positive semi-definite.

The minimal degeneracy means that critical subsets can be as degenerate as a minimum of a function, but not worse.

Remark 1.6. The condition (C3) can be reformulated as follows. Let $p: \nu\left(\Sigma_{C}\right) \rightarrow$ $\Sigma_{C}$ be the normal bundle to $\Sigma_{C}$ in $M$. Fix a Euclidian metric on $\nu\left(\Sigma_{C}\right)$ and let $|y|$ denote the norm of a vector $y \in \nu\left(\Sigma_{C}\right)$ with respect to this metric. Then by the generalized Morse lemma [21, Ch. 6] the condition (C3) is equivalent to the 
existence of a neighborhood $\mathcal{W}$ of the zero section in $\nu\left(\Sigma_{C}\right)$ and of an embedding $i: \mathcal{W} \rightarrow M$ such that

$$
\left(h_{C} \circ i\right)(y)=h_{C}(p(y))-\frac{|y|^{2}}{2},
$$

and $h_{C}$ does not have critical points on $\mathcal{W} \backslash C$.

Remark 1.7. By condition (C1), for every $C \in \mathbf{C}$ there exists a neighborhood $\mathcal{U}$ of $C$ such that $\omega$ does not have critical points in $\mathcal{U} \backslash C$. Hence, it follows from (1.5) that the restriction of $h_{C}$ to $\Sigma_{C}$ also does not have critical points on $\mathcal{U} \cap \Sigma_{C}$.

1.8. The Morse counting polynomial. The dimension of the fibers of $p: \nu\left(\Sigma_{C}\right)$ $\rightarrow \Sigma_{C}$ is called the index of the critical subset $C$ and is denoted by ind $(C)$.

Let $o\left(\Sigma_{C}\right)$ be the orientation bundle of $\nu\left(\Sigma_{C}\right)$, considered as a flat line bundle over $\Sigma_{C}$. We denote by $o(C)$ the restriction of $o\left(\Sigma_{C}\right)$ to $C$. Let $\mathcal{F}$ be a flat vector bundle over $M$. Consider the twisted Poincaré polynomial of the critical subset $C$

$$
\mathcal{P}_{C, \mathcal{F}}(\lambda)=\sum_{i=0}^{\operatorname{dim} M} \lambda^{i} \operatorname{dim}_{\mathbb{C}} \check{H}^{i}\left(C, \mathcal{F}_{\left.\right|_{C}} \otimes o(C)\right),
$$

where $\check{H}^{i}\left(C, \mathcal{F}_{\left.\right|_{C}} \otimes o(C)\right)$ denotes the Čech cohomology of $C$ with coefficients in the flat vector bundle $\mathcal{F}_{\mid C} \otimes o(C)$. Define the following Morse counting polynomial

$$
\mathcal{M}_{\omega, \mathcal{F}}(\lambda)=\sum_{C \in \mathbf{C}} \lambda^{\operatorname{ind}(C)} \mathcal{P}_{C, \mathcal{F}}(\lambda)
$$

where the sum is taken over all critical subsets $C \subset \mathbf{C}$.

1.9. The generalized Novikov numbers. Assume that $\mathcal{F}$ is a complex flat finitedimensional vector bundle over $M$ and let

$$
\nabla: \Omega^{\bullet}(M, \mathcal{F}) \rightarrow \Omega^{\bullet+1}(M, \mathcal{F})
$$

denote the covariant differential on $\mathcal{F}$.

A closed 1-form $\omega \in \Omega^{1}(M)$ on $M$ with real values determines a family of connections on $\mathcal{F}$ (the Novikov deformation) parameterized by the real numbers $T \in \mathbb{R}$

$$
\nabla_{T}: \Omega^{i}(M, \mathcal{F}) \rightarrow \Omega^{i+1}(M, \mathcal{F}) ; \quad \nabla_{T}=\nabla+T e(\omega)
$$

Here $e(\omega)$ denotes the operator of exterior multiplication by $\omega$. All the connections $\nabla_{T}$ are flat, i.e., $\nabla_{T}^{2}=0$, if the form $\omega$ is closed. Hence, for any $T \in \mathbb{R}$ the pair $\left(\mathcal{F}, \nabla_{T}\right)$ is a flat vector bundle over $M$. Sometimes we will denote this flat bundle by $\mathcal{F}_{T}$ for short.

The flat bundle $\mathcal{F}_{T}$ admits the following alternative description. For $T \in \mathbb{R}$, let $\mathcal{E}_{T \omega}$ denote the flat real line bundle over $M$ with the monodromy representation $\rho_{T \omega}: \pi_{1}(M) \rightarrow \mathbb{R}^{*}$ given by the formula

$$
\rho_{T \omega}(\gamma)=\exp \left(-T \int_{\gamma} \omega\right) \in \mathbb{R}^{*}, \quad \gamma \in \pi_{1}(M) .
$$

Then $\mathcal{F}_{T}$ is isomorphic to the tensor product $\mathcal{F} \otimes \mathcal{E}_{T \omega}$.

Assume now that $\omega$ is non-degenerate in the sense of a Kirwan closed 1-form and let $U^{-}$be as in (1.3). Let $H^{\bullet}\left(M, U^{-} ; \mathcal{F}_{T}\right)$ be the relative cohomology of the pair $\left(M, U^{-}\right)$with coefficients in the flat bundle $\mathcal{F}_{T}$.

The dimension of the cohomology $H^{\bullet}\left(M, U^{-} ; \mathcal{F}_{T}\right)$ is an integer-valued function of $T \in \mathbb{R}$. This function has the following behavior: There exists a discrete subset 
$S \subset \mathbb{R}$, such that the dimension $\operatorname{dim} H^{\bullet}\left(M, U^{-} ; \mathcal{F}_{T}\right)$ is constant for $T \notin S$ (the corresponding value of the dimension we will call the background value; the corresponding value of $T$ is called generic), and for $T \in S$ the dimension of $H^{\bullet}\left(M, U^{-} ; \mathcal{F}_{T}\right)$ is greater than the background value. Cf., for example, [18, Theorem 2.8], where a more precise information for the case of general elliptic complexes is given. The subset $S$ above will be called the set of jump points.

Definition 1.10. For each $i=0,1, \ldots, n$, the background value of the dimension of the cohomology $H^{i}\left(M, U^{-} ; \mathcal{F}_{T}\right)$ is called the $i$-th generalized Novikov number and is denoted by $\beta_{i}(\omega, \mathcal{F})$.

Remark 1.11. Note that if the boundary of $M$ is empty, then $\beta_{i}(\omega, \mathcal{F})$ are the usual Novikov numbers [23, 24, 25] with coefficients in $\mathcal{F}$; cf. [7, 10]. In this case $\beta_{i}(\omega, \mathcal{F})$ depends only on the cohomology class of $\omega$. More generally, let $\omega_{1}$ and $\omega_{2}$ be closed 1-forms representing the same cohomology class in $H^{1}(M, \mathbb{C})$ and satisfying the assumptions (B1)-(B3) of Subsection 1.2, Let $U_{1}^{-}, U_{2}^{-}$be the sets defined by (1.3) using the forms $\omega_{1}$ and $\omega_{2}$, respectively. If the topological pairs $\left(M, U_{1}^{-}\right)$and $\left(M, U_{2}^{-}\right)$are homotopically equivalent, then $\beta_{i}\left(\omega_{1}, \mathcal{F}\right)=\beta_{i}\left(\omega_{2}, \mathcal{F}\right)$.

We define the Novikov-type polynomial by the formula

$$
\mathcal{N}_{\omega, \mathcal{F}}(\lambda)=\sum_{i=0}^{\operatorname{dim} M} \lambda^{i} \beta_{i}(\omega, \mathcal{F})
$$

The main result of this paper is the following Morse-type inequality for a differential 1-form on a manifold with boundary.

Theorem 1.12. Assume that $\omega$ is a closed 1-form on $M$ which satisfies the assumptions (B1)-(B3) of Subsection 1.2 and all whose zeros are non-degenerate in the sense of Kirwan; cf. Subsection 1.4. Then there exists a polynomial $\mathcal{Q}(\lambda)=$ $q_{0}+q_{1} \lambda+q_{2} \lambda^{2}+\ldots$ with non-negative integer coefficients $q_{i} \geq 0$, such that

$$
\mathcal{M}_{\omega, \mathcal{F}}(\lambda)-\mathcal{N}_{\omega, \mathcal{F}}(\lambda)=(1+\lambda) \mathcal{Q}(\lambda) .
$$

The proof of the theorem occupies most of the remaining part of the paper.

Remark 1.13. Another version of Morse-type inequalities for differential 1-forms on manifolds with boundary were suggested by M. Farber and the first author in [8]. The conditions on the behaviour of $\omega$ near the boundary were, however, quite different in [8]. In this sense Theorem 1.12 complements the results of [8]. Note also that in [8] the zeros of the form were assumed to be non-degenerate in the sense of Bott, while in Theorem 1.12 we allow a much more complicated structure of the set of zeros.

1.14. Corollaries and applications. We will now discuss some special cases of Theorem 1.12, First, consider the case when the boundary of $M$ is empty. Then $\beta_{i}(\omega, \mathcal{F})$ are the usual Novikov numbers with coefficients in $\mathcal{F}$ associated to the cohomology class of $\omega$; cf. [10. Theorem 1.12 in this case generalizes the classical Novikov inequalities in two directions: first, we allow non-isolated zeros of $\omega$ (assuming they are non-degenerate in the sense of Kirwan). Second, we strengthen the inequalities by twisting them with the vector bundle $\mathcal{F}$ (cf. example 1.7 in [10, which shows that the obtained inequalities are really stronger than the classical inequalities with coefficients in $\mathbb{C}$. We also refer to [1, where the Kirwan inequalities with coefficients in a non-trivial bundle are used to obtain topological information 
about the symplectic reduction of a Hamiltonian $T$-space). For the case when the zeros of $\omega$ are non-degenerate manifolds (i.e., when $\omega$ satisfies Bott non-degeneracy conditions), these results were obtained by M. Farber and the first author in [7, 10].

Let us return to the case when $M$ has boundary. Assume that the cohomology class of $\omega$ vanishes, i.e., there exists a function $h: M \rightarrow \mathbb{R}$ such that $\omega=d h$. Then $\beta_{i}(\omega, \mathcal{F})$ are just the dimensions of the cohomology of the pair $\left(M, U^{-}\right)$with coefficients in $\mathcal{F}$. Thus our result generalizes the classical Morse inequalities to the case of a manifold with boundary. For the special case when $h$ is a Morse function (i.e., has only isolated non-degenerate critical points) and $\mathcal{F}=\mathbb{C}$ is the trivial bundle, similar inequalities were obtained by Floer [19].

In Section 2 we use Theorem 1.12 to obtain the equivariant version of the KirwanNovikov inequalities on a manifold with boundary. Note that the use of cohomology with coefficients in a flat vector bundle $\mathcal{F}$ (rather than just complex values cohomology) is very important for this application; cf. Remark 2.7 for details.

1.15. The necessity of condition (B3). We finish this section with a very simple example, which shows that without assumption (B3) (cf. Subsection 1.2) Theorem 1.12 is not valid. Set

Consider the cylinder $M=S^{1} \times[1,2]$. Then $\Gamma=\partial M=\left(S^{1} \times\{1\}\right) \sqcup\left(S^{1} \times\{2\}\right)$.

$$
h(x, t)=t^{2} \sin x,
$$

where $x \in S^{1}, t \in[1,2]$. Let $\omega=d h$. One easily checks that all the conditions of Theorem 1.12 except (B3) hold. Condition (B3) fails at the points $(0,1)$ and $(\pi, 1) \underline{2}$

Clearly,

$$
U^{-}=((0, \pi) \times\{1\}) \sqcup((\pi, 2 \pi) \times\{2\}) .
$$

Let $\mathcal{F}=\mathbb{C}$ be the trivial line bundle. Since the form $\omega$ is exact, $\beta_{i}=\beta_{i}(\omega, \mathcal{F})$ equals the dimension of the relative cohomology $H^{i}\left(M, U^{-}\right)$. An easy calculation shows that

$$
\beta_{0}=\beta_{2}=0, \beta_{1}=2 \text {. }
$$

Therefore, $\mathcal{N}_{\omega, \mathcal{F}}(\lambda)=2 \lambda$ in this case, but $\omega$ does not have zeros on $M$. Hence, the Morse counting polynomial is equal to 0 , and the left-hand side of (1.11) equals $-2 \lambda$. Thus (1.11) cannot hold.

Let us enlarge the manifold $M$ by setting $M^{\prime}=S^{1} \times[-2,2]$. The form $\omega=d h$ extends naturally to the bigger manifold. Now condition (B3) is easily seen to be satisfied. We still have $\mathcal{N}_{\omega, \mathcal{F}}(\lambda)=2 \lambda$, but now $\omega$ vanishes on $S^{1} \times\{0\} \subset S^{1} \times[-2,2]$. The Morse counting polynomial equals $2 \lambda$, and (1.11) holds with $\mathcal{Q}=0$.

This example illustrates an important phenomenon to be discussed in the next section: condition (B3) allows a natural extension of $\omega$ from $\mathcal{U}=\Gamma \times(0,1)$ to the infinite cylinder $\Gamma \times(0, \infty)$ without producing new zeros of $\omega$.

\footnotetext{
${ }^{2}$ Though (1.12) formally looks like the function in Example 1.3 the situation here is quite different. This is because near $S^{1} \times\{1\}$ the parameter $t$ in 1.12 is not the same as in Subsection 1.1
} 


\section{Equivariant KirWan-Novikov inequalities ON A MANIFOLD WITH BOUNDARY}

In this section we present a generalization of the equivariant Novikov inequalities obtained by M. Farber and the first author in [8, 9] to manifolds with boundary and to 1-forms which are not non-degenerate in the sense of Bott (but are nondegenerate in the sense of Kirwan). The constructions and the proofs are very similar to 9, so we will be very brief in this section and will refer the reader to 9 ] for details.

Throughout this section $G$ is a compact Lie group and $M$ is a compact $G$ manifold with boundary $\Gamma=\partial M$.

2.1. Basic 1-forms. Recall that a smooth 1 -form $\omega$ on a $G$-manifold $M$ is called basic (cf. [3]) if it is $G$-invariant and its restriction on any orbit of the action of $G$ equals zero.

Note that, if the group $G$ is finite, then $\omega$ is basic if and only if it is $G$-invariant, i.e., if $g^{*} \omega=\omega$ is satisfied for every $g \in G$. Also, if $M$ is connected and if the set of fixed points of the action of $G$ on $M$ is not empty, then any closed $G$-invariant 1-form on $M$ is basic; cf. Lemma 3.4 of [9]. Note also that any exact invariant form $\omega=d f$ is basic.

2.2. The equivariant Novikov numbers. Let $\mathcal{F} \rightarrow M$ be a $G$-equivariant flat vector bundle over $M$; cf. Section 2 of 9 . Suppose $\omega$ is a closed basic 1 -form on $M$ which satisfies assumptions (B1)-(B3) of Subsection 1.2, Let $U^{-} \subset \Gamma$ be as in (1.3). We denote by $H_{C}^{\bullet}\left(M, U^{-} ; \mathcal{F}\right)$ the equivariant cohomology of the pair $\left(M, U^{-}\right)$with coefficients in $\mathcal{F} 3$

Every closed basic 1-form $\omega$ determines an equivariant flat line bundle $\mathcal{E}_{\omega}$ (with $\omega$ being its connection form); cf. Subsection [1.9 (see also $\S 2.2$ of [9]). Using these constructions we define the equivariant Novikov numbers as follows.

Given an equivariant flat bundle $\mathcal{F}$ over $M$, consider the one-parameter family $\mathcal{F}_{T}=\mathcal{F} \otimes \mathcal{E}_{T \omega}$ of equivariant flat bundles, where $T \in \mathbb{R}$ (the Novikov deformation), and consider the twisted equivariant cohomology

$$
H_{G}^{i}\left(M, U^{-} ; \mathcal{F} \otimes \mathcal{E}_{T \omega}\right), \quad \text { where } \quad t \in \mathbb{R},
$$

as a function of $T \in \mathbb{R}$. The same arguments as in the proof of Lemma 1.5 of 9 ] show that, for each $i=0,1, \ldots$, there exists a finite subset $S \subset \mathbb{R}$ such that the dimension of the cohomology $H_{G}^{i}\left(M, U^{-} ; \mathcal{F} \otimes \mathcal{E}_{T \omega}\right)$ is constant for $T \notin S$ and the dimension of the cohomology $H_{G}^{i}\left(M, U^{-} ; \mathcal{F} \otimes \mathcal{E}_{T \omega}\right)$ jumps up for $T \in S$.

The subset $S$ is called the set of jump points; the value of the dimension of $H_{G}^{i}\left(M, U^{-} ; \mathcal{F} \otimes \mathcal{E}_{T \omega}\right)$ for $T \notin S$ is called the background value of the dimension of this family.

Definition 2.3. The $i$-dimensional equivariant Novikov number $\beta_{i}^{G}(\omega, \mathcal{F})$ is defined as the background value of the dimension of the cohomology of the family $H_{G}^{i}\left(M, U^{-} ; \mathcal{F} \otimes \mathcal{E}_{T \omega}\right)$.

Remark 2.4. If the boundary of $M$ is empty, then the numbers $\beta_{i}^{G}(\omega, \mathcal{F})$ coincide with the equivariant Novikov numbers with coefficients in $\mathcal{F}$ defined in [9. In

\footnotetext{
${ }^{3}$ The equivariant cohomology $H_{G}^{\bullet}(M ; \mathcal{F})$ with coefficients in an equivariant flat bundle are defined, for example, in $\S 2$ of [9]. The relative equivariant cohomology with coefficients is defined in exactly the same way.
} 
this case $\beta_{i}^{G}(\omega, \mathcal{F})$ depends only on the cohomology class of $\omega$. More generally, $\beta_{i}\left(\omega_{1}, \mathcal{F}\right)=\beta_{i}\left(\omega_{2}, \mathcal{F}\right)$ if $\left[\omega_{1}\right]=\left[\omega_{2}\right] \in H_{G}^{1}(M)$, and the topological pairs $\left(M, U_{1}^{-}\right)$ and $\left(M, U_{2}^{-}\right)$are homotopically equivalent (here $U_{1}^{-}, U_{2}^{-} \in \Gamma$ are the sets defined by (1.3) using the forms $\omega_{1}$ and $\omega_{2}$, respectively).

The formal power series

$$
\mathcal{N}_{\omega, \mathcal{F}}^{G}(\lambda)=\sum_{i=0}^{\infty} \lambda^{i} \beta_{i}^{G}(\omega, \mathcal{F})
$$

is called the equivariant Novikov series.

2.5. The equivariant Morse series. We assume that $\omega$ is non-degenerate in the sense of Kirwan, and we use the notation introduced in Subsections 1.4 and 1.8 . Let $C \subset \mathbf{C}$ be a component of the critical set of $\omega$. If the group $G$ is connected, then $C$ is a $G$-invariant subset of $M$. In general case we denote by

$$
G_{C}:=\{g \in G: g \cdot C \subset C\}
$$

the stabilizer of $C$ in $G$. Let $\left|G: G_{C}\right|$ denote the index of $G_{C}$ as a subgroup of $G$. Since $G_{C}$ contains the connected component of the unity in $G$, this index is finite.

The compact Lie group $G_{C}$ acts on $C$, and the flat vector bundles $\mathcal{F}_{\left.\right|_{C}}$ and $o(C)$ (cf. Subsection 1.8) are $G_{C}$-equivariant. Let $\check{H}_{G_{C}}^{*}\left(C, \mathcal{F}_{\left.\right|_{C}} \otimes o(C)\right)$ denote the equivariant $\check{C}$ ech cohomology of the flat $G_{C}$-equivariant vector bundle $\mathcal{F}_{\left.\right|_{C}} \otimes o(C)$. Consider the equivariant Poincaré series of $C$

$$
\mathcal{P}_{C, \mathcal{F}}^{G_{C}}(\lambda)=\sum_{i=0}^{\infty} \lambda^{i} \operatorname{dim}_{\mathbb{C}} H_{G_{C}}^{i}\left(C, \mathcal{F}_{\left.\right|_{C}} \otimes o(C)\right)
$$

and using it define the following equivariant Morse counting series

$$
\mathcal{M}_{\omega, \mathcal{F}}^{G}(\lambda)=\sum_{C} \lambda^{\operatorname{ind}(C)}\left|G: G_{C}\right|^{-1} \mathcal{P}_{C, \mathcal{F}}^{G_{C}}(\lambda),
$$

where the sum is taken over all components $C$ of $\mathbf{C}$.

The main result of this section is the following.

Theorem 2.6. Suppose that $G$ is a compact Lie group and $\mathcal{F}$ is a flat $G$-equivariant vector bundle over a compact $G$-manifold $M$ with boundary. Let $\omega$ be a closed basic 1-form on $M$ which satisfies the assumptions (B1)-(B3) of Subsection 1.2 and all whose zeros are non-degenerate in the sense of Kirwan; cf. Subsection 1.4. Then there exists a formal power series $\mathcal{Q}(\lambda)$ with non-negative integer coefficients, such that

$$
\mathcal{M}_{\omega, \mathcal{F}}^{G}(\lambda)-\mathcal{N}_{\xi, \mathcal{F}}^{G}(\lambda)=(1+\lambda) \mathcal{Q}(\lambda) .
$$

The theorem follows from Theorem 1.12 by exactly the same arguments as were used in [9] to derive the equivariant Novikov inequalities for a non-degenerate in the sense of a Bott one form on a closed manifold from the Novikov-Bott inequalities of [10]. We leave the details to the interested reader.

Remark 2.7. Let us remark that the use of the cohomology twisted by a flat vector bundle $\mathcal{F}$ considerably strengthens the inequalities of Theorem 2.6. For example, assume that the group $G$ is finite and the form $\omega$ is exact. An application of the equivariant Morse inequalities of Atiyah and Bott [2, 6] leads to estimates which are often weaker than the standard Morse inequalities (ignoring the group 
action). However, the application of the inequalities twisted by an equivariant flat bundle leads to much better estimates; cf. [8. This was used in [1, where an application of Theorem 2.6 to a finite group but with a non-trivial equivariant vector bundle provided new topological information about the cohomology of the symplectic reduction of a Hamiltonian $T$-manifold.

\section{Reformulation in terms of A MANifold WiTH A CYLINDRICAL END}

In this section we reformulate Theorem 1.12 as Morse-type inequalities for differential forms on a manifold with a cylindrical end. This new formulation is more suitable for our analytical proof, but also has an independent interest. In particular, it generalizes the Morse inequalities for generating functions quadratic at infinity, which are used in the theory of Lagrangian intersections [29, 16].

3.1. A manifold with a cylindrical end. By a manifold with a cylindrical end we understand a smooth (non-compact) manifold $\widetilde{M}$ without boundary which has the form $\Gamma \times(1, \infty)$ near infinity. More precisely, we suppose that there exists a compact submanifold $M \subset \widetilde{M}$ with smooth boundary $\Gamma$ such that

$$
\widetilde{M}=M \cup(\Gamma \times[1, \infty)),
$$

with $\partial M$ being identified with $\Gamma \times\{1\}$.

The submanifold $\mathcal{T}=\Gamma \times(1, \infty)$ is called the cylindrical end of $M$.

Of course, $M$ is not unique. For example, it may be replaced by $M_{t_{0}}=M \cup$ $\left(\Gamma \times\left(1, t_{0}\right]\right)$. Similarly, the decomposition $\mathcal{T}$ into a direct product is not unique. But we will fix $M$ and the decomposition $\mathcal{T}=\Gamma \times(1, \infty)$ for the sake of simplicity of the notation.

3.2. Homogeneous 1-forms. Let $\widetilde{M}=M \cup(\Gamma \times[1, \infty))$ be a manifold with a cylindrical end. Every point of $\mathcal{T}=\Gamma \times(1, \infty)$ may be identified with a pair $(x, t)$, where $x \in \Gamma, t>1$.

Let $\tau_{s}: \mathcal{T} \rightarrow \mathcal{T}(s \geq 1)$ denote the multiplication by $s: \tau_{s}(x, t)=(x, s \cdot t)$.

A differential 1-form $\alpha$ on $M$ is called homogeneous of degree $m$ at infinity if there exists $t_{0}>1$ such that

$$
\tau_{s}^{*}\left(\alpha_{\left.\right|_{\Gamma \times\left(t_{0}, \infty\right)}}\right)=s^{m} \alpha_{\left.\right|_{\Gamma \times\left(t_{0}, \infty\right)}}, \quad \text { for every } \quad s>1 .
$$

If $\omega$ is a homogeneous 1 -form of degree $m$, then its restriction to $\Gamma \times\left(t_{0}, \infty\right)$ may be written as

$$
\alpha_{\left.\right|_{\Gamma \times\left(t_{0}, \infty\right)}}=t^{m} a+f(x) t^{m-1} d t
$$

where $a$ is a 1 -form on $\Gamma$. If, in addition, $\alpha$ is closed, $d \alpha=0$, then $a=\frac{1}{m} d f$ and

$$
\alpha_{\left.\right|_{\Gamma \times\left(t_{0}, \infty\right)}}=d\left(\frac{1}{m} f(x) t^{m}\right) .
$$

In particular, the restriction of a closed homogeneous at infinity 1-form to the cylindrical part $\mathcal{T}$ is always exact.

3.3. Non-degeneracy at infinity. Suppose that $\omega$ is a closed 1-form on $\widetilde{M}$ which is homogeneous at infinity. We say that $\omega$ is non-degenerate at infinity if the restriction $\omega(x, t)$ of $\omega$ to the cylindrical part $\mathcal{T}$ does not have zeros for $t \gg 1$.

Let $\omega$ be represented as in (3.3). Then $\omega$ is non-degenerate at infinity if and only if 0 is a regular value of the function $f: \Gamma \rightarrow \mathbb{R}$. 
3.4. Example: A vector bundle over a compact manifold. One of the most important examples of a manifold with a cylindrical end is a vector bundle over a compact manifold.

Let $C$ be a smooth compact manifold without boundary and let $E$ be a finitedimensional vector bundle over $C$. Fix a Hermitian metric $h^{E}$ on $E$. For a vector $y \in E$ we denote by $|y|$ its norm with respect to the metric $h^{E}$.

Fix $r>0$ and define

$$
B_{r}=\{y \in \mathcal{E}:|y| \leq r\} ; \quad S_{r}=\{y \in \mathcal{E}:|y|=r\} .
$$

Then $B_{r}$ is a compact manifold, the boundary of $B_{r}$ equals $S_{r}$, and $E \backslash B_{r}$ is diffeomorphic to the product $S_{r} \times(1, \infty)$. Hence, $E$ has a natural structure of a manifold with a cylindrical end.

Assume now that the bundle $E$ splits into an orthogonal direct sum $E=E^{+} \oplus$ $E^{-}$. Consider the function

$$
h(y)=\frac{\left|y^{+}\right|^{2}}{2}-\frac{\left|y^{-}\right|^{2}}{2}, \quad\left(y^{+}, y^{-}\right) \in E=E^{+} \oplus E^{-} .
$$

Then the form $\omega=d h$ is homogenous of degree 2 on $E$ and is non-degenerate at infinity.

More generally, if $\widetilde{V}$ is a manifold with a cylindrical end and $\widetilde{N} \rightarrow \widetilde{V}$ is a vector bundle over $\widetilde{V}$, then one can introduce a structure of a manifold with a cylindrical end on the total space of $\widetilde{N}$. A version of this example will allow us to consider the normal bundle to a neighborhood of the critical set $C$ in the minimizing manifold $\Sigma_{C}$ (cf. Definition 1.5) as a manifold with a cylindrical end. We refer to Subsection 6.1 for details.

3.5. The Novikov numbers. Let $\omega$ be a closed 1-form on $\widetilde{M}$ which is homogeneous and non-degenerate at infinity. Let $f(x)$ be as in (3.3).

Let $\widetilde{\mathcal{F}}$ be a flat vector bundle over $\widetilde{M}$. As in Subsection 1.9 we construct a family $\widetilde{\mathcal{F}}_{T}(T \in \mathbb{R})$ of flat vector bundles over $\widetilde{M}$ using the 1 -form $\omega$.

For every $c>0$ define

$$
\widetilde{U}_{c}^{-}=\left\{(x, t) \in \Gamma \times(1, \infty): f(x) t^{m} / m<-c\right\},
$$

and consider the pair $\left(\widetilde{M}, \widetilde{U}_{c}^{-}\right)$. It is clear, that if we change $c$, then this pair will be replaced by a homotopic one. Thus the cohomology $H^{i}\left(\widetilde{M}, \widetilde{U}_{c}^{-} ; \widetilde{\mathcal{F}}_{T}\right)$ of this pair with coefficients in $\widetilde{\mathcal{F}}_{T}$ is independent of the choice of $c$. We define the Novikov number $\beta_{i}(\omega, \widetilde{\mathcal{F}})$ to be the background value of the dimension of this cohomology, cf. Subsection 1.9, and we define the Novikov polynomial $\mathcal{N}_{\omega, \widetilde{\mathcal{F}}}(\lambda)$ by (1.10).

If all the zeros of $\omega$ are non-degenerate in the sense of Kirwan we define the Morse counting polynomial $\mathcal{M}_{\omega, \widetilde{\mathcal{F}}}(\lambda)$ as in Subsection 1.8 ,

Theorem 3.6. Let $\widetilde{M}$ be a manifold with cylindrical end and let $\widetilde{\mathcal{F}}$ be a flat vector bundle over $\widetilde{M}$. Let $\omega$ be a closed 1-form on $\widetilde{M}$ which is homogeneous and nondegenerate at infinity. Assume also that all the zeros of $\omega$ are non-degenerate in the sense of Kirwan. Then there exists a polynomial $\mathcal{Q}(\lambda)$ with non-negative integer coefficients such that the Morse-type inequalities (1.11) hold.

The following proposition shows that this theorem is equivalent to Theorem 1.12 , 
Proposition 3.7. Theorem 3.6 is equivalent to Theorem 1.12 . Moreover, Theorem 3.6 (and, hence, Theorem 1.12) follows from its particular case when $\omega$ is homogeneous of degree 2 .

Thus, to prove Theorems 1.12 and 3.6. it is enough to prove Theorem 3.6 for the case when $\omega$ is homogeneous of degree 2. This will be done in the subsequent sections. The rest of this section is occupied with the proof of Proposition 3.7.

3.8. Theorem 1.12 implies Theorem 3.6. Let $\omega$ be a homogeneous, nondegenerate at infinity closed 1 -form on a manifold $\widetilde{M}$ with cylindrical end.

Fix a decomposition (3.1) of $\widetilde{M}$ and let $t_{0} \geq 0$ be as in (3.2). Set

$$
M^{\prime}=M \sqcup\left(\Gamma \times\left(1, t_{0}+2\right]\right) .
$$

Introducing a new coordinate $t^{\prime}=t-t_{0}-1$ we identify a neighborhood $\mathcal{U}$ of $\partial M^{\prime}$ with the product $\Gamma \times(0,1]$. From (3.3) we conclude that on $\mathcal{U}$ the form $\omega=d h\left(x, t^{\prime}\right)$ where

$$
h\left(x, t^{\prime}\right)=\frac{1}{m} f(x)\left(t^{\prime}+t_{0}+1\right)^{m} .
$$

As in Subsection 1.2 set

$$
U^{-}=\left\{\left(x, t^{\prime}\right): \frac{\partial h}{\partial t^{\prime}}(x, 1)<0, t^{\prime}=1\right\}=\left\{\left(x, t^{\prime}\right): f(x)<0, t^{\prime}=1\right\} .
$$

Recall that $\widetilde{U}_{c}^{-}$is defined in (3.4). Clearly, the pair $\left(\widetilde{M}, \widetilde{U}_{c}^{-}\right)$is homotopic to the pair $\left(M^{\prime}, U^{-}\right)$. Thus the relative cohomology $H^{\bullet}\left(\widetilde{M}, \widetilde{U}_{c}^{-} ; \widetilde{\mathcal{F}}_{T}\right)$ is naturally isomorphic to $H^{\bullet}\left(M, U^{-} ;\left.\widetilde{\mathcal{F}}_{T}\right|_{M^{\prime}}\right)$. Theorem 3.6 now follows from application of Theorem 1.12 to the form $\left.\omega\right|_{M^{\prime}}$.

To show the implication in the other direction we need the following

Lemma 3.9. Let $h: \Gamma \times(0,1] \rightarrow \mathbb{R}$ be a smooth function satisfying conditions (B1)-(B3) of Subsection 1.2, According to condition (B1) of Subsection 1.2, dh $\neq 0$ on $\Gamma \times(0,1]$; cf. Subsection 1.2 , Set

$$
f(x)=\frac{\partial h}{\partial t}(x, 1)
$$

Then there exist $\varepsilon \in(0,1 / 3), m>0$ and a function $\widetilde{h}: \Gamma \times(0, \infty) \rightarrow \mathbb{R}$ such that

$$
\begin{aligned}
\widetilde{h}(x, t)=h(x, t) & \text { for } t<1-2 \varepsilon, x \in \Gamma ; \\
\widetilde{h}(x, t)=m f(x) \frac{t^{2}}{2} & \text { for } t>1-\varepsilon, x \in \Gamma ; \\
d \widetilde{h}(x, t) \neq 0 & \text { for all } t>0, x \in \Gamma .
\end{aligned}
$$

Proof. From condition (B3) of Subsection 1.2 we see that there exist $\varepsilon \in(0,1 / 3)$ such that for all $t \in(1-3 \varepsilon, 1]$ we have

$$
\begin{gathered}
d_{\Gamma} h(x, t) \neq-\lambda d_{\Gamma} f(x) \quad \text { for all } \lambda>0 \text { and all } x \in \Gamma \text { such that }|f(x)|<2 \varepsilon \\
\frac{\partial h(x, t)}{\partial t} \neq-f(x) \text { for all } x \in \Gamma \text { such that }|f(x)|>\varepsilon
\end{gathered}
$$

$$
m=\frac{18}{\varepsilon} \sup \{|h(x, t)|: x \in \Gamma, 0<t \leq 1\}
$$


Let $\chi: \mathbb{R} \rightarrow[0,1]$ be a smooth non-decreasing function such that $\chi(t)=0$ for $t \leq 1-2 \varepsilon$ and $\chi(t)=1$ for $t \geq 1-\varepsilon$. Set

$$
\widetilde{h}(x, t)=(1-\chi(t)) h(x, t)+\chi(t) m f(x) \frac{t^{2}}{2} .
$$

Clearly, $\widetilde{h}$ satisfies (3.6) and (3.7). We claim that it also satisfies (3.8). Indeed,

- If $0<t<1-2 \varepsilon$, then $\widetilde{h}(x, t)=h(x, t)$, and (3.8) is exactly our assumption on $h$.

- If $1-2 \varepsilon \leq t \leq 1$ and $|f(x)|<2 \varepsilon$, then, by (3.9),

$$
d_{\Gamma} \widetilde{h}(x, t)=(1-\chi(t)) d_{\Gamma} h(x, t)+\chi(t) \frac{m t^{2}}{2} d_{\Gamma} f(x) \neq 0 .
$$

- If $1-2 \varepsilon \leq t \leq 1$ and $|f(x)|>\varepsilon$, then

$$
\frac{\partial \widetilde{h}(x, t)}{\partial t}=\chi^{\prime}(t)\left(m f(x) \frac{t^{2}}{2}-h(x, t)\right)+(1-\chi(t)) \frac{\partial h(x, t)}{\partial t}+\chi(t) m f(x) t .
$$

By (3.10) and (3.11) all the terms on the right-hand side of this equality have the same sign as $f$. Hence, $\partial \widetilde{h} / \partial t \neq 0$.

- If $t \geq 1-\varepsilon$, then $h(x, t)=m f(x) t^{2} / 2$. By assumption (B2) of Subsection 1.2, zero is a regular value of $f(x)=\frac{\partial h}{\partial t}(x, 1)$. Hence,

$$
d h(x, t)=m f(x) t d t+\frac{m t^{2}}{2} d_{\Gamma} f(x) \neq 0 .
$$

The proof of the lemma is complete.

3.10. Theorem 3.6 implies Theorem 1.12, We are ready now to show that Theorem 1.12 follows from the special case of Theorem 3.6 when $\omega$ is homogeneous of degree 2 at infinity. This will finish the proof of Proposition 3.7

Let $M, \mathcal{F}$ and $\omega$ be as in Theorem 1.12, Consider the manifold

$$
\widetilde{M}=M \cup(\Gamma \times[1, \infty))
$$

with a cylindrical end.

Fix a smooth diffeomorphism $s:(0, \infty) \rightarrow(0,1)$ such that $s(t)=t$ for $t \in$ $(0,1 / 3]$. Define a map $\bar{s}: \widetilde{M} \rightarrow M$ such that $\bar{s}(m)=m$ for $m \in \widetilde{M} \backslash(\Gamma \times[1 / 3, \infty))$ and $\bar{s}(x, t)=(x, s(t))$ for $x \in \Gamma, t \in[1 / 3, \infty)$.

Let $\widetilde{\mathcal{F}}=\bar{s}^{*} \mathcal{F}$ be the pull-back of the bundle $\mathcal{F}$ to $\widetilde{M}$. We endow $\widetilde{\mathcal{F}}$ with the pull-back connection $\bar{s}^{*} \nabla$.

Let $h(x, t)$ be as in Subsection 1.1. Assume, in addition, that $d h \neq 0$ on $\Gamma \times$ $(0,1]$, cf. Subsection 1.2, Let $\widetilde{h}$ be as in Lemma 3.9. Let $\widetilde{\omega} \in \Omega^{1}(\widetilde{M})$ be the 1form whose restriction to $\widetilde{M} \backslash(\Gamma \times(1-2 \varepsilon, \infty))$ is equal to $\omega$ and whose restriction to $\Gamma \times(1-2 \varepsilon, \infty)$ equals $d \widetilde{h}(x, t)$. From (3.7) and (3.8) we conclude that $\widetilde{\omega}$ is homogeneous of degree 2 and non-degenerate at infinity. Also, by (3.6), the forms $\widetilde{\omega}$ and $\omega$ coincide in a neighborhood of their common set of zeros. Hence their Morse counting polynomials coincide, $\mathcal{M}_{\widetilde{\omega}, \widetilde{\mathcal{F}}}(\lambda)=\mathcal{M}_{\omega, \mathcal{F}}(\lambda)$. The same arguments as in Subsection 3.8 shows that $\mathcal{N}_{\widetilde{\omega}, \widetilde{\mathcal{F}}}(\lambda)=\mathcal{N}_{\omega, \mathcal{F}}(\lambda)$. Thus the Morse-type inequalities (1.11) for $\omega$ follow from application of Theorem 3.6 to the form $\widetilde{\omega}$. The proof of Proposition 3.7 is complete. 


\section{The Witten Deformation of the Laplacian}

Let $\widetilde{M}=M \cup(\Gamma \times[1, \infty))$ be a manifold with a cylindrical end and let $\omega$ be a closed 1-form on $\widetilde{M}$ which is homogeneous of degree 2 at infinity; cf. Subsection 3.2. Let $\widetilde{\mathcal{F}}$ be a flat vector bundle over $\widetilde{M}$ endowed with a flat connection $\nabla$.

In this section we introduce a "conical at infinity" metric on $\widetilde{M}$ and use it to construct the Laplacian $\Delta_{T}$ associated to $\nabla_{T}$. This Laplacian plays a crucial role in our proof of Theorem 1.12. In Section [5, we prove that the dimension of the kernel of the restriction of $\Delta_{T}$ to the space of $p$-forms is equal to the generalized Novikov number $\beta_{p}(\xi, \widetilde{\mathcal{F}})$. In Section $\left[\right.$, we estimate the dimension of $\operatorname{Ker} \Delta_{T}$ by comparing it with the kernel of a certain Laplacian on a neighborhood of $\mathbf{C}$. That will prove Theorem 3.6 (and, hence, in view of Proposition 3.7. Theorem 1.12).

4.1. Metrics on the cylindrical end. The tangent bundle $T \mathcal{T}$ to the cylindrical end $\mathcal{T}=\Gamma \times(1, \infty)$ splits into the Whitney sum $T \Gamma \oplus \mathbb{R}$ of the tangent bundle $T \Gamma$ to $\Gamma$ and the tangent bundle to the ray $(1, \infty)$ (which we identify with $\mathbb{R}$ ).

Choose a Riemannian metric $g^{\Gamma}$ on $\Gamma$ and let $g^{\mathbb{R}}$ denote the standard (transitionally invariant) metric on $\mathbb{R}$. Let $g^{\mathcal{T}}$ denote the metric on $\mathcal{T}$ given by the formula

$$
g^{\mathcal{T}}=t^{2} g^{\Gamma} \oplus g^{\mathbb{R}} .
$$

This metric is homogeneous of degree 2 in the following sense: Let $\tau_{s}: \mathcal{T} \rightarrow \mathcal{T}$ $(s \geq 1)$ denote the multiplication by $s: \tau_{s}(x, t)=(x, s \cdot t)$. Then

$$
\tau_{s}^{*} g^{\mathcal{T}}=s^{2} g^{\mathcal{T}}
$$

We will refer to $g^{\mathcal{T}}$ as a conical metric on $\mathcal{T}$.

We also fix a Hermitian metric $h^{\left.\widetilde{\mathcal{F}}\right|_{\mathcal{T}}}$ on the restriction $\left.\widetilde{\mathcal{F}}\right|_{\mathcal{T}}$ of the bundle $\widetilde{\mathcal{F}}$ to $\mathcal{T}$ which is flat along the ray $\{(x, t): t>1\} \subset \mathcal{T}$ for any $x \in \Gamma$.

4.2. Metrics on the manifold. Let $g^{\widetilde{M}}$ be a Riemannian metric on $\widetilde{M}$ whose restriction on the cylindrical end $\mathcal{T}$ is equal to the conical metric $g^{\mathcal{T}}$ (cf. Subsection 4.1).

We also fix a Hermitian metric $h^{\widetilde{\mathcal{F}}}$ on $\widetilde{\mathcal{F}}$ whose restriction to $\mathcal{T}$ is equal to $\left.h^{\tilde{\mathcal{F}}}\right|_{\mathcal{T}}$.

4.3. The deformation of the Laplacian. Let $\nabla_{T}=\nabla+T e(\omega)$ be the Novikov deformation of the covariant derivative; cf. Subsection 1.9, Denote by $\nabla_{T}^{*}$ the formal adjoint of $\nabla_{T}$ with respect to the metrics $g^{\widetilde{M}}, h^{\widetilde{\mathcal{F}}}$.

Definition 4.4. The Witten Laplacian is the operator

$$
\Delta_{T}:=\frac{1}{T}\left(\nabla_{T} \nabla_{T}^{*}+\nabla_{T}^{*} \nabla_{T}\right): \Omega^{\bullet}(\widetilde{M}, \widetilde{\mathcal{F}}) \rightarrow \Omega^{\bullet}(\widetilde{M}, \widetilde{\mathcal{F}}) .
$$

It is well known (cf., for example, [12, [20, Th. 1.17], [11) that the operator $\Delta_{T}$ is essentially self-adjoint with initial domain smooth compactly supported forms. By a slight abuse of notation we will also denote by $\Delta_{T}$ the self-adjoint extension of this operator to the space $L^{2} \Omega^{\bullet}(\widetilde{M}, \widetilde{\mathcal{F}})$ of square-integrable forms.

Proposition 4.5. For every $T>0$ the spectrum of the operator $\Delta_{T}$ is discrete.

We prove the proposition in Subsection 4.8 after we study the behaviour of the restriction of $\Delta_{T}$ to the cylindrical end $\mathcal{T}$. 
4.6. The restriction of the deformed Laplacian to the cylindrical end. Let $v$ denote the vector field on $\widetilde{M}$ which corresponds to the 1 -form $\omega$ via the Riemannian metric $g^{\widetilde{M}}$. Since $\omega$ is homogeneous of degree 2, it follows immediately from (4.2) that the restriction of $v$ to $\mathcal{T}$ is homogeneous of degree 0 , i.e., is independent of $t$ :

$$
v(x, s t)=\tau_{s *} v(x, t), \quad \text { for all } s>1 .
$$

We denote by $\mathcal{L}_{v}$ the Lie derivative along $v$. An easy calculation (cf. [14, Proposition 11.13]) shows that

$$
\Delta_{T}=\frac{1}{T} \Delta+\left(\mathcal{L}_{v}+\mathcal{L}_{v}^{*}\right)+T|\omega|^{2} .
$$

Here $\mathcal{L}_{v}^{*}$ is the formal adjoint of $\mathcal{L}_{v}$ and $\Delta=\nabla \nabla^{*}+\nabla^{*} \nabla$ stands for the usual Laplacian on $\widetilde{\mathcal{F}}$. The operators $\left(\mathcal{L}_{v}+\mathcal{L}_{v}^{*}\right)$ and $T|\omega|^{2}$ are symmetric zero order differential operators, i.e., self-adjoint endomorphisms of the vector bundle $\Lambda^{\bullet}\left(T^{*} \widetilde{M}\right) \otimes \widetilde{\mathcal{F}}$ of $\widetilde{\mathcal{F}}$-valued forms on $\widetilde{M}$.

We consider now the restriction of (4.4) to the cylindrical end $\mathcal{T}$. For every $x \in \Gamma$, the flat structure defines a trivialization of $\widetilde{\mathcal{F}}\left(\right.$ and, hence, of $\left.\Lambda^{\bullet}\left(T^{*} \widetilde{M}\right) \otimes \widetilde{\mathcal{F}}\right)$ along the ray $\{(x, t) \in \mathcal{T}: t>1\}$. Thus for every $x \in \Gamma$ we can consider

$$
a(x, t):=\left(\mathcal{L}_{v}+\mathcal{L}_{v}^{*}\right) \quad \text { and } \quad b(x, t):=|\omega(x, t)|^{2}
$$

as a function of $t$ with values in the space of symmetric matrices.

The proof of Proposition 4.5 is based on the following simple lemma, which follows immediately from (4.2), (4.3), and the fact that $\omega$ is homogeneous of degree 2 and non-degenerate at infinity:

Lemma 4.7. For all $s>1, x \in \Gamma, t \in[1, \infty)$ the following equalities hold:

$$
a(x, s t)=a(x, t), \quad b(x, s t)=s^{2} b(x, t)>0 .
$$

4.8. Proof of Proposition 4.5. It is well known (cf., for example, 28, Lemma 6.3]) that Proposition 4.5 is equivalent to the following statement: For every $\varepsilon>0$ there exists a compact set $K \subset \widetilde{M}$ such that, if $\alpha \in \Omega^{\bullet}(\widetilde{M}, \widetilde{\mathcal{F}})$ is a smooth compactly supported form, then

$$
\int_{\widetilde{M} \backslash K}|\alpha|^{2} d \mu<\varepsilon \int_{\widetilde{W}}\left\langle\Delta_{T} \alpha, \alpha\right\rangle d \mu .
$$

Here, $d \mu$ is the Riemannian volume element on $\widetilde{M}$, and $\langle\cdot, \cdot\rangle$ denotes the Hermitian scalar product on the fibers of $\Lambda^{\bullet}\left(T^{*} \widetilde{M}\right) \otimes \widetilde{\mathcal{F}}$ induced by the Riemannian metric on $\widetilde{M}$ and the Hermitian metric on $\widetilde{\mathcal{F}}$.

From (4.6), we conclude that there exists $t_{0}>1$ such that

$$
a(x, t)+b(x, t)>1 / \varepsilon, \quad \text { for all } \quad x \in \Gamma, t \geq t_{0} .
$$

Then, using (4.4) and (4.5), we obtain

$$
\int_{\Gamma \times\left[t_{0}, \infty\right)}|\alpha|^{2} d \mu<\varepsilon \int_{\Gamma \times\left[t_{0}, \infty\right)}\langle(a+b) \alpha, \alpha\rangle d \mu \leq \varepsilon \int_{\Gamma \times\left[t_{0}, \infty\right)}\left\langle\Delta_{T} \alpha, \alpha\right\rangle d \mu .
$$


Theorem 4.9. For every $T>0$, the dimension of the kernel Ker $\Delta_{T}$ of $\Delta_{T}$ is equal to the dimension of the relative cohomology $H^{\bullet}\left(\widetilde{M}, \widetilde{U}^{-} ; \widetilde{\mathcal{F}}_{T}\right)$.

In particular, $\operatorname{dim} \operatorname{Ker} \Delta_{T}^{i}=\beta_{i}(\omega, \mathcal{F})$ for a generic value of $T$.

The prove of the theorem occupies the next section of the paper.

\section{Computation of the kernel of the Witten Laplacian}

In this section we prove Theorem 4.9 .

5.1. Complex $\widetilde{\Omega}_{T}^{\bullet}(\widetilde{M}, \widetilde{F})$. The kernel of the Witten Laplacian $\operatorname{Ker} \Delta_{T}$ has the following cohomological interpretation. Define

$$
\widetilde{\Omega}_{T}^{\bullet}(\widetilde{M}, \widetilde{\mathcal{F}})=\left\{\zeta \in L^{2} \Omega^{\bullet}(\widetilde{M}, \widetilde{\mathcal{F}}): \nabla_{T} \zeta \in L^{2} \Omega^{\bullet}(\widetilde{M}, \widetilde{\mathcal{F}})\right\}
$$

and consider the complex

$$
0 \rightarrow \widetilde{\Omega}_{T}^{0}(\widetilde{M}, \widetilde{\mathcal{F}}) \stackrel{\nabla_{T}}{\longrightarrow} \widetilde{\Omega}_{T}^{1}(\widetilde{M}, \widetilde{\mathcal{F}}) \stackrel{\nabla_{T}}{\longrightarrow} \cdots \stackrel{\nabla_{T}}{\longrightarrow} \widetilde{\Omega}_{T}^{n}(\widetilde{M}, \widetilde{\mathcal{F}}) \rightarrow 0
$$

Let $H^{\bullet}\left(\widetilde{\Omega_{T}}(\widetilde{M}, \widetilde{\mathcal{F}}), \nabla_{T}\right)$ denote the cohomology of this complex.

Proposition 5.2. $H^{k}\left(\widetilde{\Omega}_{T}^{\bullet}(\widetilde{M}, \widetilde{\mathcal{F}}), \nabla_{T}\right)=\operatorname{dim} \operatorname{Ker} \Delta_{T}^{k}$ for all $k=0,1, \ldots, n$.

Proof. Since the spectrum of $\Delta_{T}$ is discrete (cf. Proposition 4.5), the space $L^{2} \Omega^{\bullet}(\widetilde{M}, \widetilde{\mathcal{F}})$ splits into the orthogonal direct sum of closed subspaces

$$
L^{2} \Omega^{\bullet}(\widetilde{M}, \widetilde{\mathcal{F}})=\operatorname{Ker} \Delta_{T} \oplus \operatorname{Im} \Delta_{T}
$$

Hence, every $\varkappa \in \widetilde{\Omega}_{T}^{\bullet}(\widetilde{M}, \widetilde{\mathcal{F}})$ can be represented as the sum

$$
\varkappa=\varphi+\Delta_{T} \psi=\varphi+\frac{1}{T} \nabla_{T} \nabla_{T}^{*} \psi+\frac{1}{T} \nabla_{T}^{*} \nabla_{T} \psi,
$$

where $\varphi \in \operatorname{Ker} \Delta_{T}$ and $\psi \in \operatorname{Dom}\left(\Delta_{T}\right) \subset L^{2} \Omega^{\bullet}(\widetilde{M}, \widetilde{\mathcal{F}})$. Since $\nabla_{T}^{2}=0$ all 3 summands on the right-hand side of (5.2) are mutually orthogonal and belong to $L^{2} \Omega^{\bullet}(\widetilde{M}, \widetilde{\mathcal{F}})$.

The ellipticity of $\Delta_{T}$ implies that the forms $\phi$ and $\psi$ are smooth. Hence, so are the forms $\nabla_{T} \psi, \nabla_{T}^{*} \psi, \nabla_{T} \nabla_{T}^{*} \psi$, and $\nabla_{T}^{*} \nabla_{T} \psi$. Moreover, $\nabla_{T} \psi, \nabla_{T}^{*} \psi \in$ $L^{2} \Omega^{\bullet}(\widetilde{M}, \widetilde{\mathcal{F}})$ because of the inequality

$$
\left\|\nabla_{T} \psi\right\|^{2}+\left\|\nabla_{T}^{*} \psi\right\|^{2}=T\left\langle\Delta_{T} \psi, \psi\right\rangle<\infty
$$

(here $\langle\cdot, \cdot\rangle$ and $\|\cdot\|$ stand for the scalar product and the norm in $L^{2} \Omega^{\bullet}(\widetilde{M}, \widetilde{\mathcal{F}})$, respectively). We conclude that $\nabla_{T} \psi, \nabla_{T}^{*} \psi \in \widetilde{\Omega}_{T}^{\bullet}(\widetilde{M}, \widetilde{\mathcal{F}})$. Hence, (5.2) implies that the following "Hodge-type" decomposition holds:

$$
\widetilde{\Omega}_{T}^{\bullet}(\widetilde{M}, \widetilde{\mathcal{F}})=\operatorname{Ker} \Delta_{T} \oplus \operatorname{Im}\left(\left.\nabla_{T}\right|_{\widetilde{\Omega}_{T}^{\bullet}(\widetilde{M}, \widetilde{\mathcal{F}})}\right) \oplus \operatorname{Im}\left(\left.\nabla_{T}^{*}\right|_{\widetilde{\Omega}_{T}(\widetilde{M}, \widetilde{\mathcal{F}})}\right) .
$$

The statement of the proposition now follows from the standard "Hodge theory" arguments.

Theorem 4.9 now follows from the following proposition.

Proposition 5.3. The cohomology of the complex (5.1) is isomorphic to the relative cohomology $H^{\bullet}\left(\widetilde{M}, \widetilde{U}^{-} ; \widetilde{\mathcal{F}}_{T}\right)$.

The proof of the proposition occupies the rest of this section. 
5.4. The cone complex. Let $c$ be an arbitrary positive constant and let $\widetilde{U}_{c}^{-}$be as in (3.4). We denote by $j_{c}: \widetilde{U}_{c}^{-} \rightarrow \widetilde{M}$ the inclusion and by $j_{c}^{*}: \Omega^{\bullet}(\widetilde{M}, \stackrel{\mathcal{F}}{)} \rightarrow$ $\Omega^{\bullet}\left(\widetilde{U}_{c}^{-},\left.\widetilde{\mathcal{F}}\right|_{\widetilde{U}_{c}^{-}}\right)$the restriction map.

Consider the cone complex $\mathrm{Cone}_{T}\left(j_{c}^{*}\right)$ of the map $j_{c}^{*}$ which is defined as follows:

$$
\operatorname{Cone}_{T}^{k}\left(j_{c}^{*}\right)=\Omega^{k}(\widetilde{M}, \widetilde{\mathcal{F}}) \oplus \Omega^{k-1}\left(\widetilde{U}_{c}^{-}, \widetilde{\mathcal{F}}\right)
$$

and the differential $D_{T, c}:$ Cone $_{T}^{k}\left(j_{c}^{*}\right) \rightarrow$ Cone $_{T}^{k+1}\left(j_{c}^{*}\right)$ is given by

$$
D_{T, c}\left(\eta, \eta_{1}\right)=\left(\nabla_{T} \eta,-\nabla_{T} \eta_{1}+j_{c}^{*} \eta\right), \quad\left(\eta, \eta_{1}\right) \in \mathrm{Cone}_{T}^{\bullet}\left(j_{c}^{*}\right) .
$$

It is well known (see, e.g., [15]) that

$$
H^{\bullet}\left(\operatorname{Cone}_{T}\left(j_{c}^{*}\right)\right) \cong H^{\bullet}\left(\widetilde{M}, \widetilde{U}_{c}^{-} ; \widetilde{\mathcal{F}}_{T}\right) .
$$

Thus, to prove Proposition 5.3 (and, hence, Theorem 4.9) it suffices to show that the space $H^{\bullet}\left(\operatorname{Cone}_{T}\left(j_{c}^{*}\right)\right)$ is isomorphic to the cohomology $H^{\bullet}\left(\widetilde{\Omega}_{T}^{\bullet}(\widetilde{M}, \widetilde{\mathcal{F}}), \nabla_{T}\right)$ of the complex (5.1). This is done in the next subsection, where we construct an explicit map from $\widetilde{\Omega}_{T}^{\bullet}(\widetilde{M}, \widetilde{\mathcal{F}})$ to $\operatorname{Cone}_{T}\left(j_{c}^{*}\right)$ and show that this map is a quasi-isomorphism (i.e., induces an isomorphism on cohomology). A similar construction was used by Farber and Shustin [17, §3] in a slightly different situation.

5.5. A map from $\widetilde{\Omega}_{T}^{\bullet}(\widetilde{M}, \widetilde{F})$ to $\operatorname{Cone}_{T}\left(j_{c}^{*}\right)$. Recall that we assumed that $\omega$ is homogeneous of degree 2 and non-degenerate at infinity. Thus (cf. (3.3) ) there exist $t_{0}>0$ and a smooth function $f: \Gamma \rightarrow \mathbb{R}$ such that

$$
\omega(x, t)=d\left(f(x) t^{2} / 2\right), \quad x \in \Gamma, t \geq t_{0},
$$

and 0 is a regular value of $f$. Changing the parameter $t$ if needed, we can and we will assume that $t_{0}=1$. Set

$$
h(x, t)=f(x) t^{2} / 2 .
$$

The restriction of any form $\zeta \in \Omega^{\bullet}(\widetilde{M}, \widetilde{\mathcal{F}})$ to the cylindrical end $\mathcal{T}$ can be written as

$$
\zeta(x, t)=d t \wedge \zeta_{\|}(x, t)+\zeta_{\perp}(x, t), \quad x \in \Gamma, \quad t>1,
$$

where $\zeta_{\|}(x, t)$ and $\zeta_{\perp}(x, t)$ are $\widetilde{\mathcal{F}}$-valued forms on $\Gamma$ depending smoothly on the parameter $t \in(0, \infty)$. Set

$$
\begin{aligned}
\breve{\zeta}(x, t)=-e^{-T h(x, t)} & \int_{t}^{\infty} e^{T h(x, \tau)} \zeta_{\|}(x, \tau) d \tau \\
& =-\int_{t}^{\infty} e^{T f(x)\left(\tau^{2}-t^{2}\right) / 2} \zeta_{\|}(x, \tau) d \tau, \quad(x, t) \in \widetilde{U}_{c}^{-},
\end{aligned}
$$

where we use the flat connection $\nabla$ to identify the fibers of $\widetilde{\mathcal{F}}$ along the rays $\{(x, \tau): \tau>0\}$. The integral in (5.6) converges since $\zeta \in L^{2} \Omega^{\bullet}\left(\widetilde{U}_{c}^{-}, \widetilde{\mathcal{F}}\right)$ and $f(x)<0$ for $(x, t) \in \widetilde{U}_{c}^{-}$.

Define the map $\Phi_{T, c}: \widetilde{\Omega}_{T}^{\bullet}(\widetilde{M}, \widetilde{\mathcal{F}}) \rightarrow \operatorname{Cone}_{T}\left(j_{c}^{*}\right)$ by the formula

$$
\Phi_{T, c}: \zeta \mapsto(\zeta, \breve{\zeta})
$$


Lemma 5.6. For every $c>0$ the map $\Phi_{T, c}$ is a chain map from $\widetilde{\Omega}_{T}^{\bullet}\left(\widetilde{M}, \widetilde{\mathcal{F}} ; \nabla_{T}\right)$ to Cone $_{T}\left(j_{c}^{*}\right)$, i.e.,

$$
D_{T, c} \circ \Phi_{T, c}=\Phi_{T, c} \circ \nabla_{T} .
$$

Proof. Let $\zeta \in \widetilde{\Omega}_{T}^{\bullet}(\widetilde{M}, \widetilde{\mathcal{F}})$. The equality (5.8) reduces to

$$
\begin{aligned}
\nabla_{T}\left[e^{-T h(x, t)} \int_{t}^{\infty} e^{T h(x, \tau)} \zeta_{\|}(x, \tau) d \tau\right] & +\zeta(x, t) \\
= & -e^{-T h(x, t)} \int_{t}^{\infty} e^{T h(x, \tau)}\left(\nabla_{T} \zeta(x, \tau)\right)_{\|} d \tau
\end{aligned}
$$

for $(x, t) \in \widetilde{U}_{c^{\prime}}^{-}$. The last equality follows from the following calculation, in which we denote by $\nabla_{\Gamma}$ the connection induced by $\nabla$ on $\Gamma$ and by $\nabla_{\partial / \partial \tau}$ the covariant differential along the vector field $\partial / \partial \tau$ :

$$
\begin{aligned}
& \nabla_{T}\left[e^{-T h} \int_{t}^{\infty} e^{T h} \zeta_{\|} d \tau\right]+\zeta=e^{-T h} \nabla \int_{t}^{\infty} e^{T h} \zeta_{\|} d \tau+\zeta \\
& =-d t \wedge \zeta_{\|}+e^{-T h} \int_{t}^{\infty} \nabla_{\Gamma}\left(e^{T h} \zeta_{\|}\right) d \tau+\zeta=\zeta_{\perp}+e^{-T h} \int_{t}^{\infty} \nabla_{\Gamma}\left(e^{T h} \zeta_{\|}\right) d \tau \\
& =e^{-T h} \int_{t}^{\infty}\left(\nabla_{\Gamma}\left(e^{T h} \zeta_{\|}\right)-\nabla_{\partial / \partial \tau}\left(e^{T h} \zeta_{\perp}\right)\right) d \tau=-e^{-T h} \int_{t}^{\infty} e^{T h}\left(\nabla_{T} \zeta\right)_{\|} d \tau
\end{aligned}
$$

Let

$$
\Phi_{T, c, *}: H^{\bullet}\left(\widetilde{\Omega} \widetilde{\Omega}_{T}^{\bullet}(\widetilde{M}, \widetilde{\mathcal{F}}), \nabla_{T}\right) \longrightarrow H^{\bullet}\left(\operatorname{Cone}_{T}\left(j_{c}^{*}\right)\right) \cong H^{\bullet}\left(\widetilde{M}, \widetilde{U}_{c}^{-} ; \widetilde{\mathcal{F}}_{T}\right)
$$

be the map induced by $\Phi_{T, c}$. To prove Proposition 5.3 we need now to show that the map $\Phi_{T, c, *}$ is an isomorphism.

5.7. Injectivity of $\Phi_{T, c, *}$. The proof of the injectivity of $\Phi_{T, c, *}$ is based on the following technical lemma.

Lemma 5.8. Let $\zeta \in \widetilde{\Omega}_{T}^{\bullet}(\widetilde{M}, \widetilde{\mathcal{F}})$ be a "harmonic" form, $\Delta_{T} \zeta=0$. Suppose that

$$
D_{T, c}\left(\alpha, \alpha_{1}\right)=\Phi_{T, c} \zeta, \quad \text { for some }\left(\alpha, \alpha_{1}\right) \in \operatorname{Cone}_{T}\left(j_{c}^{*}\right) .
$$

Then there exist $\varepsilon>0$ and a form $\beta \in \Omega^{\bullet}(\widetilde{M}, \widetilde{\mathcal{F}})$ such that

(i) $\nabla_{T} \beta=\zeta$;

(ii) $\beta(x, t)=\breve{\zeta}(x, t)=-\int_{t}^{\infty} e^{\frac{1}{2} T f(x)\left(\tau^{2}-t^{2}\right)} \zeta_{\|}(x, \tau) d \tau$, for $x \in \mathcal{S}_{\varepsilon}, t>3$;

(iii) $\beta_{\|}(x, t)=0$ for $x \in \Gamma, t>3$.

Moreover, any such form $\beta$ belongs to $\widetilde{\Omega}_{T}^{\bullet}(\widetilde{M}, \widetilde{\mathcal{F}})$.

Proof. We will construct the form $\beta$ by "improving" the form $\alpha$ in 3 steps.

Step 1. Fix $c^{\prime}>c$ large enough, so that $\widetilde{U}_{c^{\prime}}^{-} \subset \Gamma \times(3,+\infty)$. Choose a smooth function $\chi_{1}: \widetilde{M} \rightarrow[0,1]$ such that $\chi_{1} \equiv 1$ on $\widetilde{U}_{c^{\prime}}^{-}$and $\chi_{1} \equiv 0$ on $\widetilde{M} \backslash \widetilde{U}_{c}^{-}$. Our first approximation of $\beta$ is the form

$$
\alpha^{\prime}=\alpha-\nabla_{T}\left(\chi_{1} \alpha_{1}\right)
$$


From (5.9) and (5.10) we obtain

$$
\begin{array}{cc}
\nabla_{T} \alpha^{\prime}=\zeta & \text { on } \widetilde{M} ; \\
\alpha^{\prime}=\breve{\zeta} & \text { on } \widetilde{U}_{c^{\prime}}^{-} .
\end{array}
$$

On the cylindrical end $\mathcal{T}$ we can write $\alpha^{\prime}=d t \wedge \alpha_{\|}^{\prime}+\alpha_{\perp}^{\prime}$; cf. (5.5). Then the equation (5.12) implies that

$$
\alpha_{\|}^{\prime}=0 \quad \text { on } \widetilde{U}_{c^{\prime}}^{-} .
$$

Step 2. Take a new smooth cut-off function $\chi_{2}: \mathbb{R} \rightarrow[0,1]$, such that $\chi_{2}(t)=0$ if $t \leq 1$ and $\chi_{2}(t)=1$ if $t \geq 2$, and set

$$
\alpha^{\prime \prime}(x, t)=\alpha^{\prime}(x, t)-\nabla_{T}\left(\chi_{2}(t) e^{-T h(x, t)} \int_{2}^{t} e^{T h(x, \tau)} \alpha_{\|}^{\prime}(x, \tau) d \tau\right) .
$$

Again we have $\nabla_{T} \alpha^{\prime \prime}=\zeta$. Since $\chi_{2}(t) \equiv 0$ for $t \geq 2$, we also have

$$
\alpha_{\|}^{\prime \prime}=0 \quad \text { on } \Gamma \times(2, \infty) \text {, }
$$

so that

$$
\nabla_{T} \alpha_{\perp}^{\prime \prime}=\zeta \quad \text { on } \quad \Gamma \times(2, \infty)
$$

In particular,

$$
e^{-T h(x, t)} \nabla_{\partial / \partial t} e^{T h(x, t)} \alpha_{\perp}^{\prime \prime}(x, t)=\zeta_{\|}(x, t), \quad x \in \Gamma, t>2 .
$$

Step 3. For each $r \in \mathbb{R}$ denote

$$
\mathcal{S}_{r}=\{x \in \Gamma: f(x)<r / T\} .
$$

Recall that we assumed that $\Delta_{T} \zeta=0$. A verbatim repetition of the arguments in [27. Appendix] shows that on the cylindrical part $\mathcal{T}$ the form $\zeta$ satisfies the estimate

$$
|\zeta(x, t)|<C e^{-a t^{2}}
$$

with some positive constants $C$ and $a$. (Here $|\zeta(x, t)|$ denotes the norm of $\zeta(x, t)$ with respect to the "conical" Riemannian metric introduced in Subsection 4.1.) Hence,

$$
\left|e^{T h(x, t)} \zeta_{\|}(x, t)\right| \leq e^{-(a-r / 2) t^{2}}, \quad \text { if } f(x)<r / T,
$$

i.e., $e^{T h} \zeta_{\|}$decays exponentially when $t$ tends to infinity and $x \in \mathcal{S}_{2 a}$. Thus the solution of the ordinary differential equation (5.17) can be written in the form

$$
e^{T h(x, t)} \alpha_{\perp}^{\prime \prime}(x, t)=\eta(x)-\int_{t}^{\infty} e^{T h(x, \tau)} \zeta_{\|}(x, \tau) d \tau, \quad x \in \mathcal{S}_{2 a}, t>2,
$$

where $\eta(x)$ is a differential form on $S_{2 a} \subset \Gamma$ (independent of $t$ ).

From (5.16) and (5.20) we see that on $\mathcal{S}_{2 a} \times(2, \infty)$

$$
\zeta_{\perp}=e^{-T h} \nabla_{\Gamma} e^{T h} \alpha_{\perp}^{\prime \prime}=e^{-T h} \nabla_{\Gamma} \eta-e^{-T h} \int_{t}^{\infty} \nabla_{\Gamma} e^{T h} \zeta_{\|} d \tau .
$$

Since $\nabla_{T} \zeta=0$ we have

$$
0=\left(\nabla_{T} \zeta\right)_{\|}=-e^{-T h} \nabla_{\Gamma} e^{T h} \zeta_{\|}+e^{-T h} \nabla_{\partial / \partial t} e^{T h} \zeta_{\perp},
$$

i.e.,

$$
\nabla_{\Gamma} e^{T h} \zeta_{\|}=\nabla_{\partial / \partial t} e^{T h} \zeta_{\perp} .
$$


Substituting this equality into (5.21), we see that on $\mathcal{S}_{2 a} \times(2, \infty)$

$$
\zeta_{\perp}=e^{-T h} \nabla_{\Gamma} \eta-e^{-T h} \int_{t}^{\infty} \nabla_{\partial / \partial \tau} e^{T h} \zeta_{\perp} d \tau=e^{-T h} \nabla_{\Gamma} \eta+\zeta_{\perp} .
$$

Hence,

$$
\nabla_{\Gamma} \eta(x)=0, \quad \text { if } \quad x \in \mathcal{S}_{2 a},
$$

i.e., $\eta$ is a closed form on $\mathcal{S}_{2 a}$.

Set $\gamma=\int_{2}^{\infty} e^{T h} \alpha_{\|}^{\prime} d \tau$. Comparing (5.20) with (5.14) and using (5.12) we see that $\eta=\nabla \gamma$ on $\widetilde{U}_{c^{\prime}}^{-}$. Since, by the construction, $\gamma$ is independent of $t$, we obtain $\eta=\nabla_{\Gamma} \gamma$ on $\widetilde{U}_{c^{\prime}}^{-}$. But, for all $x \in \mathcal{S}_{0}$, there exists $t$ such that $(x, t) \in \widetilde{U}_{c^{\prime}}^{-}$. Therefore, $\eta=\nabla_{\Gamma} \gamma$ on $\mathcal{S}_{0}$. In particular, the restriction of $\eta$ to $\mathcal{S}_{0}$ represents the zero class in $H^{\bullet}\left(\mathcal{S}_{0}\right)$.

Since 0 is a regular value of $f$ there exists $\varepsilon \in(0, a)$ such that $\mathcal{S}_{0}$ is a deformation retract of $\mathcal{S}_{2 \varepsilon}$. Hence $\eta$ also represents the trivial class in $H^{\bullet}\left(S_{2 \varepsilon}\right)$, i.e., there exists a form $\xi \in \Omega^{\bullet}\left(\mathcal{S}_{2 \varepsilon}\right)$ such that

$$
\nabla_{\Gamma} \xi(x)=\eta(x), \quad x \in \mathcal{S}_{2 \varepsilon} .
$$

Consider a smooth cut-off function $\chi_{3}: \Gamma \rightarrow[0,1]$, such that $\chi_{3}=1$ on $\mathcal{S}_{\frac{4}{3} \varepsilon}$ and $\chi_{3}=0$ on $\Gamma \backslash \mathcal{S}_{\frac{5}{3} \varepsilon}$. Finally, set

$$
\beta=\alpha^{\prime \prime}-\nabla_{T}\left(e^{-T h(x, t)} \chi_{3}(x) \chi_{2}(t) \xi\right) .
$$

Since $\beta$ is obtained by subtracting exact forms from $\alpha$, condition (i) of the lemma is satisfied. From (5.15), (5.22), (5.23), and (5.20) we obtain

$$
\beta(x, t)=\alpha_{\perp}^{\prime \prime}(x, t)-e^{-T h(x, t)} \eta(x)=-\int_{t}^{\infty} e^{\frac{1}{2} T f(x)\left(\tau^{2}-t^{2}\right)} \zeta_{\|}(x, \tau) d \tau,
$$

for $x \in \mathcal{S}_{\varepsilon}, t>3$. Thus condition (ii) of the lemma is satisfied.

On $\Gamma \times(3,+\infty)$ form $\beta$ is obtained from $\alpha^{\prime \prime}$, whose parallel component vanishes, by subtracting $\nabla_{T}\left(e^{-T h(x, t)} \chi_{3}(x) \xi\right)$, which also does not have a parallel component. Therefore, condition (iii) of the lemma is also satisfied.

It now remains to prove that $\beta$ is in $\widetilde{\Omega}_{T}^{\bullet}(\widetilde{M}, \widetilde{\mathcal{F}})$. The estimate (5.19) implies that $\nabla_{T} \beta=\zeta$ is in $L^{2} \Omega^{\bullet}(\widetilde{M}, \widetilde{\mathcal{F}})$, so we only need to show that $\beta \in L^{2} \Omega(\widetilde{M}, \widetilde{\mathcal{F}})$. Since the form $\beta$ is smooth, its restriction to every compact set is square integrable. Hence, it is enough to show that the restrictions of $\beta$ to $\mathcal{S}_{\varepsilon} \times(3, \infty)$ and $\left(\Gamma \backslash \mathcal{S}_{\varepsilon}\right) \times(3, \infty)$ are square integrable.

Restriction of $\beta$ to $\mathcal{S}_{\varepsilon} \times(3, \infty)$. Using the estimate (5.19), we see that on $\mathcal{S}_{\varepsilon} \times(3, \infty)$

$$
|\beta(x, t)|=\left|\int_{t}^{\infty} e^{\frac{1}{2} T f(x)\left(\tau^{2}-t^{2}\right)} \zeta_{\|}(x, \tau) d \tau\right| \leq C \int_{t}^{\infty} e^{-(a-\varepsilon / 2) \tau^{2}} d \tau .
$$

Since $\varepsilon<a$ we conclude that $\beta$ is decaying exponentially with $t$ and, hence, is square integrable on $\mathcal{S}_{\varepsilon} \times(3, \infty)$.

Restriction of $\beta$ to $\left(\Gamma \backslash S_{\varepsilon}\right) \times(3, \infty)$. Since $\beta_{\|}=0$ on $\Gamma \times(3, \infty)$, taking the parallel component of the equality $\zeta=\nabla_{T} \beta$, we obtain

$$
e^{-T h(x, t)} \nabla_{\partial / \partial t} e^{T h(x, t)} \beta(x, t)=\zeta_{\|}(x, t) .
$$


Solving this ordinary differential equation we get

$$
e^{T h(x, t)} \beta(x, t)=\beta(x, 3)+\int_{3}^{t} e^{T h(x, \tau)} \zeta_{\|}(x, \tau) d \tau .
$$

Therefore, setting $B=\max _{x \in \Gamma}|\beta(x, 3)|$ and using (5.19), we see that on $\left(\Gamma \backslash S_{\varepsilon}\right) \times(3, \infty)$ the following estimate holds:

$$
\begin{aligned}
& |\beta(x, t)| \leq B e^{-\frac{1}{2} T f(x) t^{2}}+\int_{3}^{t} e^{\frac{1}{2} T f(x)\left(\tau^{2}-t^{2}\right)}\left|\zeta_{\|}(x, \tau)\right| d \tau \\
\leq & B e^{-\varepsilon t^{2} / 2}+\int_{3}^{t} e^{\frac{1}{2} \varepsilon\left(\tau^{2}-t^{2}\right)} e^{-a \tau^{2}} d \tau \leq B e^{-\varepsilon t^{2} / 2}+e^{-\frac{1}{2} \varepsilon t^{2}} \int_{3}^{t} e^{-\left(a-\frac{1}{2} \varepsilon\right) \tau^{2}} d \tau .
\end{aligned}
$$

Thus $\beta(x, t)$ decays exponentially when $t$ tends to infinity and, hence, is square integrable on $\left(\Gamma \backslash S_{\varepsilon}\right) \times(3, \infty)$. The proof of the lemma is now complete.

Corollary 5.9. The map $\Phi_{T, c, *}$ is injective.

Proof. Let $\Upsilon \in H^{\bullet}\left(\widetilde{\Omega_{T}}(\widetilde{M}, \widetilde{\mathcal{F}})\right)$ be a cohomology class, whose image under $\Phi_{T, c, *}$ is trivial. By Proposition 5.2, the representative $\zeta \in \Upsilon$ can be chosen in such a way that $\Delta_{T} \zeta=0$. Then Lemma 5.8 implies that there exist $\beta \in \widetilde{\Omega}_{T}^{\bullet}(\widetilde{M}, \widetilde{\mathcal{F}})$, such that $\nabla_{T} \beta=\zeta$. Hence, $\Upsilon=0$.

5.10. Surjectivity of $\Phi_{T, c, *}$. In order to prove that $\Phi_{T, c, *}$ is surjective we need the following auxiliary lemma, which, roughly speaking, states that every relative cohomology class has a bounded representative.

Recall that the sets $\mathcal{S}_{r} \subset \Gamma$ were defined in (5.18). Since 0 is a regular value of $f$ we can take $\varepsilon>0$ small enough, such that for all $\varepsilon^{\prime} \in[0, \varepsilon]$, the set $\mathcal{S}_{\varepsilon^{\prime}}$ is homotopically equivalent to $\mathcal{S}_{0}=U^{-}$. For any $\delta>1$ denote by $j_{\delta, \varepsilon}$ the inclusion map of $\mathcal{S}_{\varepsilon} \times[\delta,+\infty)$ into $\widetilde{M}$. Let $\operatorname{Cone}_{T}\left(j_{\delta, \varepsilon}^{*}\right)$ be the complex

$$
\operatorname{Cone}_{T}^{k}\left(j_{\delta, \varepsilon}^{*}\right)=\Omega^{k}(\widetilde{M}, \widetilde{\mathcal{F}}) \oplus \Omega^{k-1}\left(\mathcal{S}_{\varepsilon} \times[\delta,+\infty)\right), \quad k=0, \ldots, \operatorname{dim} \widetilde{M},
$$

with the differential

$$
D_{T, \delta, \varepsilon}\left(\eta, \eta_{1}\right)=\left(\nabla_{T} \eta,-\nabla_{T} \eta_{1}+j_{c}^{*} \eta\right), \quad\left(\eta, \eta_{1}\right) \in \operatorname{Cone}_{T}^{\bullet}\left(j_{c}^{*}\right) .
$$

Also for any $\delta>0$ denote by $\widetilde{M}_{\delta}$ the compact set $M \cup(\Gamma \times[1, \delta)) \subset \widetilde{M}$.

Lemma 5.11. Every cohomological class in $H^{\bullet}\left(\mathrm{Cone}_{T}\left(j_{\delta, \varepsilon}^{*}\right)\right)$ has a representative $\left(\alpha, \alpha_{1}\right)$, such that the restrictions of the forms $e^{T h} \alpha, e^{T h} \alpha_{1}, \nabla_{T} e^{T h} \alpha$, and $\nabla_{T} e^{T h} \alpha_{1}$ to the cylindrical end $\mathcal{T}$ are bounded with respect to the Riemannian metric introduced in Subsection 4.1 .

Proof. As in the proof of Lemma 5.8 we start with an arbitrary representative $\left(\alpha^{\prime}, \alpha_{1}^{\prime}\right)$ and "improve it".

For all $\varepsilon^{\prime} \in(0, \varepsilon)$ and $\delta^{\prime}>\delta$, the pairs $\left(\widetilde{M}, \mathcal{S}_{\varepsilon} \times[\delta,+\infty)\right)$ and $\left(M_{\delta^{\prime}}, \mathcal{S}_{\varepsilon^{\prime}} \times\left[\delta, \delta^{\prime}\right)\right)$ are homotopically equivalent. Moreover, there exists a smooth map $\mathcal{R}: \widetilde{M} \rightarrow \widetilde{M}_{\delta^{\prime}}$, such that:

(i) $\mathcal{R}$ is a homotopy equivalence of the topological pairs $\left(\widetilde{M}, \mathcal{S}_{\varepsilon} \times[\delta,+\infty)\right)$ and $\left(\widetilde{M}_{\delta^{\prime}}, \mathcal{S}_{\varepsilon^{\prime}} \times\left[\delta, \delta^{\prime}\right)\right)$,

(ii) the restriction of $\mathcal{R}$ to $\widetilde{M}_{\delta}$ is the identity operator,

(iii) the tangent map $T \mathcal{R}$ is bounded (with respect to the Riemannian metric introduced in Subsection 4.1). 
On the cylindrical end $\mathcal{T}=\Gamma \times[1,+\infty)$ the differentials $\nabla_{T}$ and $D_{T, \delta, \varepsilon}$ can be written as follows:

$$
\nabla_{T}=e^{-T h} \nabla_{T} e^{T h}, \quad D_{T, \delta, \varepsilon}=e^{-T h} D_{0, \delta, \varepsilon} e^{T h} .
$$

Properties (i) and (ii) of the map $\mathcal{R}$ imply that on $\mathcal{T}$

$$
e^{T h}\left(\alpha^{\prime}, \alpha_{1}^{\prime}\right)-\mathcal{R}^{*} e^{T h}\left(\alpha^{\prime}, \alpha_{1}^{\prime}\right)=D_{0, \delta, \varepsilon}\left(\mu, \mu_{1}\right)
$$

for some $\mu \in \Omega^{\bullet}(\mathcal{T}, \widetilde{\mathcal{F}})$ and $\mu_{1} \in \Omega^{\bullet}\left(\mathcal{S}_{\varepsilon} \times[\delta,+\infty)\right)$. Let $\chi: \mathbb{R} \rightarrow[0,1]$ be a smooth non-decreasing function, such that

$$
\chi(t)=0 \text { for } t \leq \frac{1+\delta}{4}, \text { and } \quad \chi(t)=1 \text { for } t \geq \frac{1+\delta}{2} .
$$

We also denote by $\chi$ its natural extension to $\widetilde{M}$. Set

$$
\left(\alpha, \alpha_{1}\right)=\left(\alpha^{\prime}, \alpha_{1}^{\prime}\right)-D_{T, \delta, \varepsilon}\left(e^{-T h} \chi \cdot\left(\mu, \mu_{1}\right)\right) .
$$

Then $\left(\alpha, \alpha_{1}\right)$ and $\left(\alpha^{\prime}, \alpha_{1}^{\prime}\right)$ represent the same cohomological class in $\left(\operatorname{Cone}_{T}\left(j_{\delta, \varepsilon}^{*}\right)\right)$. By construction, $\chi(t)=1$ for $t \geq \frac{1+\delta}{2}$. Hence, using (5.24) and (5.25), we obtain

$$
\begin{gathered}
e^{T h}\left(\alpha, \alpha_{1}\right)=e^{T h}\left(\left(\alpha^{\prime}, \alpha_{1}^{\prime}\right)-D_{T, \delta, \varepsilon}\left(e^{-T h}\left(\mu, \mu_{1}\right)\right)\right) \\
=e^{T h}\left(\alpha^{\prime}, \alpha_{1}^{\prime}\right)-D_{0, \delta, \varepsilon}\left(\mu, \mu_{1}\right) \\
=\mathcal{R}^{*} e^{T h}\left(\alpha^{\prime}, \alpha_{1}^{\prime}\right), \quad \text { on } \quad \Gamma \times[2,+\infty) .
\end{gathered}
$$

The pair $\mathcal{R}^{*} e^{T h}\left(\alpha^{\prime}, \alpha_{1}^{\prime}\right)$ is the pull-back of the restriction of $e^{T h}\left(\alpha^{\prime}, \alpha_{1}^{\prime}\right)$ to the finite part $\widetilde{M}_{\delta^{\prime}}$ of $\widetilde{M}$. Since $T \mathcal{R}$ is bounded, (5.26) implies that $e^{T h} \alpha$ and $e^{T h} \alpha_{1}$ are also bounded together with their differentials 4

Corollary 5.12. The map $\Phi_{T, c, *}$ is surjective.

Proof. Since the topological pairs $\left(\widetilde{M}, \widetilde{U}_{c}^{-}\right)$are homotopically equivalent for different $c>0$, it suffices to prove the corollary for sufficiently large $c$. Therefore, we can assume that $c$ is large enough, so that $\widetilde{U}_{c}^{-} \subset \Gamma \times[3,+\infty)$.

Let $\Upsilon \in H^{\bullet}\left(\operatorname{Cone}_{T}\left(j_{c}^{*}\right)\right)$ be an arbitrary cohomology class. Take $\varepsilon>0$ small enough, so that $U^{-}=\mathcal{S}_{0}$ is a deformation retract of $\mathcal{S}_{\varepsilon^{\prime}}$ for all $\varepsilon^{\prime} \in[0,2 \varepsilon]$. Let

$$
R: \operatorname{Cone}_{T}\left(j_{2,2 \varepsilon}^{*}\right) \ni\left(\alpha, \alpha_{1}\right) \mapsto\left(\alpha,\left.\alpha_{1}\right|_{\widetilde{U}_{c}^{-}}\right) \in \operatorname{Cone}_{T}\left(j_{c}^{*}\right)
$$

be the restriction map. Since $\widetilde{U}_{c}^{-}$is a deformation retract of $\mathcal{S}_{2 \varepsilon} \times[2,+\infty)$, the map $R$ induces an isomorphism of cohomology. Therefore, $\Upsilon$ can be represented by a pair $R\left(\alpha, \alpha_{1}\right)$, where $\left(\alpha, \alpha_{1}\right) \in \operatorname{Cone}_{T}\left(j_{2,2 \varepsilon}^{*}\right)$. By Lemma 5.11, the pair $\left(\alpha, \alpha_{1}\right)$ can be chosen in such a way that the forms $e^{T h} \alpha, e^{T h} \alpha_{1}, e^{T h} \nabla_{T} \alpha$, and $e^{T h} \nabla_{T} \alpha_{1}$ are all bounded.

Let $\chi: \widetilde{M} \rightarrow[0,1]$ be a smooth function, such that

$$
\chi=0 \quad \text { on } \widetilde{M} \backslash\left(\mathcal{S}_{2 \varepsilon} \times[2,+\infty)\right), \quad \text { and } \quad \chi=1 \quad \text { on } \mathcal{S}_{\varepsilon} \times[3,+\infty),
$$

and $d \chi$ is bounded on $\mathcal{T}$ with respect to the Riemannian metric introduced in Subsection 4.1. Set

$$
\beta=\alpha-\nabla_{T}\left(\chi \alpha_{1}\right)
$$

\footnotetext{
${ }^{4}$ Recall that we use the conical metric on $\mathcal{T}$ introduced in Subsection 4.1 Thus, if, for example, we consider a form $\gamma \in \Omega^{\bullet}(\Gamma)$ as a constant form on $\mathcal{T}=\Gamma \times(0, \infty)$, then the norm $|\gamma|_{(x, t)}$ of $\gamma$ at the point $(x, t) \in \mathcal{T}$ decays as $1 / t$ as $t \rightarrow \infty$.
} 
Then the pair $(\beta, 0)$ is cohomologous to the pair $\left(\alpha, \alpha_{1}\right)$ in the $\mathrm{Cone}_{T}\left(j_{c}^{*}\right)$. In addition, $\beta$ vanishes on $\mathcal{S}_{\varepsilon} \times[3,+\infty) \supset \widetilde{U}_{c}^{-}$, hence $\left[\Phi_{T, c} \beta\right]=\Upsilon$. In order to finish the proof of the corollary it remains to show that $\beta \in \widetilde{\Omega}_{T}^{\bullet}(\widetilde{M}, \widetilde{\mathcal{F}})$. Since $\nabla_{T} \beta=$ $\nabla_{T} \alpha=0$, we only need to check that $\beta$ is square integrable on the cylindrical end $\mathcal{T}$. Since $\beta$ vanishes on $\mathcal{S}_{\varepsilon} \times[3,+\infty)$ we only need to consider its restriction to $\left(\Gamma \backslash \mathcal{S}_{\varepsilon}\right) \times[3,+\infty)$. The boudedness of $e^{T h} \alpha, e^{T h} \alpha_{1}$ and $d \chi$ implies that $\left|e^{T h} \beta\right|<B$ for some $B>0$. Hence, on $\left(\Gamma \backslash \mathcal{S}_{\varepsilon}\right) \times[3,+\infty)$ we have

$$
|\beta(x, t)|<e^{-T h(x, t)} B=B e^{-\frac{1}{2} f(x) t^{2}}<B e^{-\frac{1}{2} \varepsilon t^{2}},
$$

hence $\beta$ is square integrable and the map $\Phi_{T, c, *}$ is surjective.

The proof of Theorem 4.9 is complete.

\section{Deformation of the Laplacian on the normal Bundle TO A MINIMIZING MANIFOLD}

In this section for each critical subset $C$ of $\omega$ we introduce a manifold $\widetilde{N}_{C}$ with a cylindrical end, whose compact part may be identified with a neighborhood of $C$ in $\widetilde{M}$. We also introduce a family of connections $\nabla_{T}^{\widetilde{N}_{C}}$ on the lift of $\widetilde{\mathcal{F}}$ to $\widetilde{N}_{C}$, which is similar to the Novikov deformation (1.8). We apply the machinery of Section 4 to the Laplacians $\Delta_{T}^{\widetilde{N}_{C}}$ of the connections $\nabla_{T}^{\widetilde{N}_{C}}$. In particular, we show that the spectrum of $\Delta_{T}^{\widetilde{N}_{C}}$ is discrete and does not depend on $T$, and we compute the kernel of $\Delta_{T}^{\widetilde{N}_{C}}$. The dimension of the kernel $\Delta_{T}^{\widetilde{N}_{C}}$ encodes the information about the topology of the critical subset $C$; cf. Theorem 6.9.

The significance of the operators $\Delta_{T}^{\widetilde{N}_{C}}$ is that their direct sum $\bigoplus_{C \in \mathbf{C}} \Delta_{T}^{\widetilde{N}_{C}}$ plays the role of a model operator (cf. [27]) for the family $\Delta_{T}$ of Laplacian on the manifold $\widetilde{M}$. More precisely, this statement is formulated in the next section (Theorem 7.3).

6.1. Construction of the manifold $\widetilde{N}_{C}$. Let $C \subset \mathbf{C}$ be a critical subset of $\omega$; cf. Subsection 1.4. Let $h_{C}, \Sigma_{C}$ be as in Definition 1.5 and let $\nu\left(\Sigma_{C}\right), \mathcal{W} \subset \nu\left(\Sigma_{C}\right)$ be as in Remark 1.6.

Fix a Hermitian metric $h^{\nu\left(\Sigma_{C}\right)}$ and a Hermitian connection $\nabla^{\nu\left(\Sigma_{C}\right)}$ on the vector bundle $p: \nu\left(\Sigma_{C}\right) \rightarrow \Sigma_{C}$.

Set

$$
V_{C, r}:=\left\{x \in \Sigma_{C}: h_{C}(x)<r^{2}\right\} .
$$

Fix a small enough number $\varepsilon>0$ so that $V_{C, 7 \varepsilon} \subset \mathcal{W}$, the restriction of $h_{C}$ to $\Sigma_{C} \cap \mathcal{W}$ does not have critical values on the interval $(0,7 \varepsilon]$, and

$$
\left\{y \in \nu\left(\Sigma_{C}\right): \frac{|y|^{2}}{2} \leq(7 \varepsilon)^{2}, h_{C}(p(y)) \leq(7 \varepsilon)^{2}\right\} \subset \mathcal{W} .
$$

Using the gradient flow of $\left.h_{C}\right|_{\Sigma_{C}}$ with respect to some arbitrary metric, we can construct a family of smooth maps $m_{t}: V_{C, 7 \varepsilon} \rightarrow V_{C, 7 \varepsilon}(0<t \leq 1)$ such that $m_{t_{1}} \circ m_{t_{2}}=m_{t_{1} t_{2}}$ and

$$
\begin{array}{rlrl}
h_{C}\left(m_{t}(x)\right) & =t^{2} h_{C}(x), & & x \in V_{C, 7 \varepsilon} \backslash V_{C, \varepsilon / 2}, 1 / 2<t \leq 1, \\
h_{C}(x) \geq h_{C}\left(m_{t}(x)\right) \geq t^{2} h_{C}(x), & & x \in V_{C, 7 \varepsilon}, 0<t \leq 1 .
\end{array}
$$

For each $y \in p^{-1} V_{C, 7 \varepsilon}$ let

$$
\widetilde{m}_{t}(y) \in p^{-1}\left(m_{t}(p(x))\right)
$$


denote the horizontal lift of the curve $t \mapsto m_{t}(p(y))$. Then $\left|\widetilde{m}_{t}(y)\right|=|y|$ for all $t \in(0,1]$. Set

$$
y(t)=t \widetilde{m}_{t}(y) .
$$

Let

$$
F_{C}(y)=h_{C}(p(y))+\frac{|y|^{2}}{2}, \quad y \in \nu\left(\Sigma_{C}\right)
$$

and, for each $r \in[1,7]$, define

$$
\begin{aligned}
B_{C, r} & =\left\{y \in p^{-1} V_{C, 7 \varepsilon}: F_{C}(y(1 / r)) \leq \varepsilon^{2}\right\}, \\
S_{C, r} & =\left\{y \in p^{-1} V_{C, 7 \varepsilon}: F_{C}(y(1 / r))=\varepsilon^{2}\right\} .
\end{aligned}
$$

Then $B_{C, r}$ is a compact manifold with smooth boundary $S_{C, r}$. The maps $y \mapsto y(t)$ identify $B_{C, 6} \backslash B_{C, 1}$ with the product $S_{C, 1} \times(1,6)$. Hence, we can define the manifold with a cylindrical end

$$
\widetilde{N}_{C}:=B_{C, 6} \cup\left(S_{C, 1} \times(1, \infty)\right) .
$$

Note that

$$
V_{C, \varepsilon}=\left(B_{C, 1} \backslash S_{C, 1}\right) \cap \Sigma_{C}
$$

6.2. The function $\widetilde{h}_{C}$. Recall that in Remark 1.6 we defined an embedding $i$ : $\mathcal{W} \rightarrow M$. By a slight abuse of the notation we will also denote by $h_{C}$ the lift $h_{C} \circ i$ of this function to $\mathcal{W}$. Let $\widetilde{h}_{C}: \widetilde{N}_{C} \rightarrow \mathbb{R}$ be a smooth function such that

$$
\widetilde{h}_{C}(x, t)=t^{2} h_{C}(x, 1), \quad t \geq 1, x \in S_{C, 1} .
$$

By (6.2) and (1.5), we have

$$
\widetilde{h}_{C}(y)=t^{2} h_{C}\left(m_{1 / t}(p(y))\right)-\frac{|y|^{2}}{2}, \quad y=(x, t) \in B_{C, 6} \backslash B_{C, 1} .
$$

From (6.1), we now conclude that

$$
\widetilde{h}_{C}(y)=h_{C}(y) \quad \text { and } \quad d \widetilde{h}_{C}(y)=d h_{C}(y) \quad \text { for } \quad y \in\left(B_{C, 6} \backslash B_{C, 1}\right) \cap \Sigma_{C} \text {. }
$$

From (6.1) and (6.2), we see that

$$
\widetilde{h}_{C}(\tau y)<\widetilde{h}_{C}(y), \quad \text { for all } \quad \tau>1, \quad y \in\left(B_{C, 6} \backslash B_{C, 1}\right) \backslash \Sigma_{C} .
$$

The last inequality can be rewritten as

$$
\iota_{y} d \widetilde{h}_{C}(y)<0
$$

were we identify $y$ with the vertical tangent vector to $\nu\left(\Sigma_{C}\right)$ and denote by $\iota_{y}$ the interior multiplication by this vector. Combining (6.7), (6.8), and (1.5) we conclude that

$$
d \widetilde{h}_{C}(y) \neq-\lambda d h_{C}(y), \quad \text { for all } \quad \lambda>0, \quad y \in B_{C, 6} \backslash B_{C, 1} .
$$


6.3. Deformation of the covariant differential. Choose a smooth nondecreasing function $\sigma:[0,+\infty) \rightarrow[0,1]$, such that

$$
\sigma(t)=0 \quad \text { for } t \in[0,2], \quad \text { and } \quad \sigma(t)=1 \quad \text { for } t \in[3,+\infty) .
$$

For each $s>0$, set

$$
\phi(s, y)= \begin{cases}1, & \text { if } \quad y \in B_{C, 2}, \\ s^{\sigma(t)}, & \text { if } \quad y=(x, t) \in S_{C, 1} \times(2, \infty) .\end{cases}
$$

Since $\sigma$ vanishes on $[0,2]$, the function $\phi(s, y)$ is well defined and smooth on $\widetilde{N}_{C}$. The vector bundle $\widetilde{\mathcal{F}}$ induces naturally a flat vector bundle on $\widetilde{N}_{C}$, which we will also denote by $\widetilde{\mathcal{F}}$. Let $\nabla^{\widetilde{N}_{C}}$ denote the covariant differential operator determined by the flat structure on $\widetilde{\mathcal{F}}$. (We use the superscript $\widetilde{N}_{C}$ here, to distinguish this operator from the covariant differential on the bundle $\mathcal{F}$ over the manifold $\widetilde{M}$.) Consider the one-parameter deformation $\nabla_{T}^{\widetilde{N}_{C}}$ of this covariant differential defined by the formula

$$
\nabla_{T}^{\widetilde{N}_{C}}=e^{-\phi(T, y) \cdot \widetilde{h}_{C}(y)} \nabla^{\widetilde{N}_{C}} e^{\phi(T, y) \cdot \widetilde{h}_{C}(y)} .
$$

Remark 6.4. Note that, for each $T>0$, equation (6.10) is equivalent to (1.8) with

$$
\omega=\frac{1}{T} d\left(\phi(T, y) \widetilde{h}_{C}\right) .
$$

Therefore, we can apply the results of the previous sections of the paper to the connection $\nabla_{T}^{\widetilde{N}_{C}}$. Moreover, since the form $\omega$ is exact on $\widetilde{N}_{C}$, the Novikov numbers will be replaced everywhere by the usual Betti numbers.

For every $s>0$, let $r_{s}: \widetilde{N}_{C} \rightarrow \widetilde{N}_{C}$ be the map defined by

$$
r_{s}(y)= \begin{cases}y, & \text { if } y \in B_{C, 2}, \\ (x, \phi(s, y) t), & \text { if } y=(x, t) \in S_{C, 1} \times(2, \infty) .\end{cases}
$$

If $y \in \widetilde{N}_{C}$, we denote by $\widetilde{\mathcal{F}}_{y}$ the fiber of $\widetilde{\mathcal{F}}$ over $y$. The flat connection on $\widetilde{\mathcal{F}}$ gives a natural identification of the fibers $\widetilde{\mathcal{F}}_{y}$ and $\widetilde{\mathcal{F}}_{s y}$. Hence, the map $r_{s}: \widetilde{N}_{C} \rightarrow \widetilde{N}_{C}$ defines the "pull-back" map

$$
r_{s}^{*}: \Omega^{\bullet}\left(\widetilde{N}_{C}, \widetilde{\mathcal{F}}\right) \rightarrow \Omega^{\bullet}\left(\widetilde{N}_{C}, \widetilde{\mathcal{F}}\right) .
$$

Lemma 6.5. For every $T>0$

$$
\nabla_{T}^{\widetilde{N}_{C}}=r_{\sqrt{T}}^{*} e^{-\widetilde{h}_{C}} \nabla^{\widetilde{N}_{C}} e^{\widetilde{h}_{C}}\left(r_{\sqrt{T}}^{*}\right)^{-1} .
$$

Proof. Since $r_{\sqrt{T}}^{*}$ commutes with $\nabla^{\widetilde{N}_{C}}$, we can write

$$
\begin{aligned}
r_{\sqrt{T}}^{*} e^{-\widetilde{h}_{C}} \nabla e^{\widetilde{h}_{C}}\left(r_{\sqrt{T}}^{*}\right)^{-1}=r_{\sqrt{T}}^{*} e^{-\widetilde{h}_{C}}\left(r_{\sqrt{T}}^{*}\right)^{-1} \nabla_{T} r_{\sqrt{T}}^{*} e^{\widetilde{h}_{C}}\left(r_{\sqrt{T}}^{*}\right)^{-1} & \\
& =e^{-\phi(T, y) \widetilde{h}_{C}} \nabla^{\widetilde{N}_{C}} e^{\phi(T, y) \widetilde{h}_{C}}=\nabla_{T}^{\widetilde{N}_{C}} .
\end{aligned}
$$

Here the last line follows from the identity

$$
r_{\sqrt{T}}^{*} \widetilde{h}_{C}(y)\left(r_{\sqrt{T}}^{*}\right)^{-1}=\phi(T, y) \widetilde{h}_{C}(y),
$$

where $\widetilde{h}_{C}$ is identified with the operator of multiplication by $\widetilde{h}_{C}$. 
6.6. Deformation of the Laplacian. Let us choose a Riemannian metric $g_{1}^{\widetilde{N}_{C}}$ on $\widetilde{N}_{C}$ and a Hermitian metric $h_{1}^{\widetilde{\mathcal{F}}}$ on $\widetilde{\mathcal{F}}$ which on the cylindrical end $S_{C, 1} \times(1, \infty)$ have the form described in Subsection 4.1. Consider the families of metrics $g_{T}^{\widetilde{N}_{C}}$ and $h_{T}^{\widetilde{\mathcal{F}}}$ depending on $T$ given by

$$
g_{T}^{\widetilde{N}_{C}}=\frac{1}{T} r_{\sqrt{T}}^{*} g_{1}^{\widetilde{N}_{C}}, \quad h_{T}^{\widetilde{\mathcal{F}}}=r_{\sqrt{T}}^{*} h_{1}^{\widetilde{\mathcal{F}}} .
$$

Definition 6.7. The Witten Laplacian $\Delta^{\widetilde{N}_{C}}$ of the bundle $\widetilde{N}_{C}$ associated to the metrics $g_{T}^{\widetilde{N}_{C}}, h^{\widetilde{\mathcal{F}}}$ is defined by the formula

$$
\Delta_{T}^{\widetilde{N}_{C}}=\frac{1}{T}\left(\nabla_{T}^{\widetilde{N}_{C}} \nabla_{T}^{\widetilde{N}_{C}^{*}}+\nabla_{T}^{\widetilde{N}_{C}^{*}} \nabla_{T}^{\widetilde{N}_{C}}\right),
$$

where $\nabla_{T}^{\widetilde{N}_{C} *}$ denote the formal adjoint of $\nabla_{T}^{\widetilde{N}_{C}}$ with respect to the metrics $g_{T}^{\widetilde{N}_{C}}, h_{T}^{\tilde{\mathcal{F}}}$. We denote by $\Delta_{T}^{\widetilde{N}_{C}, p}$ the restriction of $\Delta_{T}^{\widetilde{N}_{C}}$ to the space of $p$-forms.

Proposition 6.8. For every $T>0$,

$$
\Delta_{T}^{\widetilde{N}_{C}}=r_{\sqrt{T}}^{*} \Delta_{1}^{\widetilde{N}_{C}}\left(r_{\sqrt{T}}^{*}\right)^{-1} .
$$

In particular, the operators $\Delta_{T}^{\widetilde{N}_{C}}$ and $\Delta_{1}^{\widetilde{N}_{C}}$ have the same spectrum.

Proof. Lemma 6.5 implies that

$$
\nabla_{T}^{\widetilde{N}_{C}}=r_{\sqrt{T}}^{*} \nabla_{1}^{\widetilde{N}_{C}}\left(r_{\sqrt{T}}^{*}\right)^{-1} .
$$

The metrics $g_{T}^{\widetilde{N}_{C}}=\frac{1}{T} r_{\sqrt{T}}^{*} g_{1}^{\widetilde{N}_{C}}$ and $h_{T}^{\widetilde{\mathcal{F}}}=r_{\sqrt{T}}^{*} h_{1}^{\widetilde{\mathcal{F}}}$ are defined in such a way that the map

$$
r_{\sqrt{T}}:\left(\widetilde{N}_{C}, T g_{T}^{\widetilde{N}_{C}}\right) \rightarrow\left(\widetilde{N}_{C}, g_{1}^{\widetilde{N}_{C}}\right)
$$

is an isometry (here $\left(\widetilde{N}_{C}, T g_{T}^{\widetilde{N}_{C}}\right)$ and $\left(\widetilde{N}_{C}, g_{1}^{N_{C}}\right)$ denote $\widetilde{N}_{C}$ considered as a Riemannian manifold with the Riemannian metric $T g_{T}^{\widetilde{N}_{C}}$ and $g_{1}^{\widetilde{N}_{C}}$, respectively). Moreover, the pull-back map

$$
r_{\sqrt{T}}^{*}: L^{2}\left(\Omega^{\bullet}\left(\widetilde{N}_{C}, \widetilde{\mathcal{F}}\right), g_{1}^{\widetilde{N}_{C}}\right) \rightarrow L^{2}\left(\Omega^{\bullet}\left(\widetilde{N}_{C}, \widetilde{\mathcal{F}}\right), T g_{T}^{\widetilde{N}_{C}}\right)
$$

is also an isometry (here $L^{2}\left(\Omega^{\bullet}\left(\widetilde{N}_{C}, \widetilde{\mathcal{F}}\right), g\right)$ denotes the space of differential forms, which are square-integrable with respect to the scalar product induced by the Riemannian metric $g$ ).

We conclude that the adjoint operator to the differential $\nabla$ is equal to

$$
\nabla_{T}^{*}=\frac{1}{T} r_{\sqrt{T}}^{*} \nabla_{1}^{*}\left(r_{\sqrt{T}}^{*}\right)^{-1}
$$

The equality (6.14) now follows from (6.15) and (6.16).

Recall from Subsection 1.8 that for each critical subset $C$, we denote by $\operatorname{ind}(C)$ the dimension of the fibers of the bundle $\nu\left(\Sigma_{C}\right) \rightarrow \Sigma_{C}$ and by $o(C)$ the orientation bundle of $\nu\left(\Sigma_{C}\right)$, considered as a flat line bundle.

Recall that $\varepsilon>0$ was chosen in Subsection 6.1. The following theorem gives the main spectral properties of the Witten Laplacian $\Delta_{T}^{\widetilde{N}_{C}}$. 
Theorem 6.9. (1) For each $T>0$ the operator $\Delta_{T}^{\widetilde{N}_{C}}$ has a discrete spectrum. More precisely, there exists an orthonormal basis of smooth eigenforms with eigenvalues $\lambda_{j} \in \mathbb{R}$, such that $\lambda_{j} \rightarrow \infty$ as $j \rightarrow \infty$. The spectrum of $\Delta_{T}^{N_{C}}$ coincides with the set of all eigenvalues $\left\{\lambda_{j}\right\}$.

(2) Let $\Delta_{T}^{\widetilde{N}_{C}, p}$ denote the restriction of $\Delta_{T}^{\widetilde{N}_{C}}$ on the space of $p$-forms. Then

$$
\operatorname{dim} \operatorname{Ker} \Delta_{T}^{\widetilde{N}_{C}, p}=\operatorname{dim} H^{p-\operatorname{ind}(C)}\left(V_{C, \varepsilon}, \mathcal{F}_{\left.\right|_{V_{C, \varepsilon}}} \otimes o\left(\Sigma_{C}\right)_{\left.\right|_{V_{C, \varepsilon}}}\right)
$$

for any $p=0,1, \ldots, \operatorname{dim} M$.

Proof. Part (1) of the theorem is a particular case of Proposition 4.5.

Since the deformation $\nabla_{T}^{\widetilde{N}_{C}}$ is defined using the exact form $\frac{1}{T} d\left(\phi \widetilde{h}_{C}\right)$ (cf. Remark 6.4), Theorem 4.9 implies that

$$
\operatorname{dim} \operatorname{Ker} \Delta_{T}^{\widetilde{N}_{C}, p}=\operatorname{dim} H^{p}\left(\widetilde{N}_{C}, \widetilde{U}_{C, c}^{-} ; \mathcal{F}\right)
$$

where $c>0$ and

$$
\widetilde{U}_{C, c}^{-}=\left\{y \in \widetilde{N}_{C}: \widetilde{h}_{C}(y)<c\right\}
$$

Set $U_{C}^{-}:=\left\{y \in S_{C, 1}: \widetilde{h}_{C}(y)<0\right\}$. Clearly, the topological pair $\left(\widetilde{N}_{C}, \widetilde{U}_{C, c}^{-}\right)$is homotopic to the pair $\left(B_{C, 1}, U_{C}^{-}\right)$.

Let $\nu_{C, \varepsilon}=p^{-1} V_{C, \varepsilon}$ be the restriction of the bundle $p: \nu\left(\Sigma_{C}\right) \rightarrow \Sigma_{C}$ to $V_{C, \varepsilon}$. From (6.6) and (6.4), we see that the pair $\left(B_{C, 1}, U_{C}^{-}\right)$is homotopic to the pair $\left(\nu_{C, \varepsilon}, \nu_{C, \varepsilon} \backslash V_{C, \varepsilon}\right)$. Hence, the right-hand side of (6.18) is isomorphic to the cohomology $H^{p}\left(\nu_{C, \varepsilon}, \nu_{C, \varepsilon} \backslash V_{C, \varepsilon} ; \mathcal{F}\right)$, which, via the Thom isomorphism, is isomorphic to the right-hand side of (6.17).

\section{Proof of the KirWan-Novikov inequalities}

In this section we prove Theorem 3.6 (and, hence, in view of Proposition 3.7, also Theorem 1.12). The main ingredient of the proof is Theorem 7.3 which estimates the spectrum of the Laplacian $\Delta_{T}$ (cf. Subsection 4.3) in terms of the spectrum of the Laplacian $\bigoplus_{C \in \mathbf{C}} \Delta_{T}^{\widetilde{N}_{C}}$ (cf. Subsection 6.6 ). We postpone the proof of Theorem 7.3 to the next section.

\subsection{The deformed Laplacian. Set}

$$
\begin{aligned}
\tilde{N} & =\bigsqcup_{C \in \mathbf{C}} \widetilde{N}_{C}, \quad B_{r}=\bigsqcup_{C \in \mathbf{C}} B_{C, r}, \quad S_{r}=\bigsqcup_{C \in \mathbf{C}} S_{C, r}, \\
g_{T}^{\widetilde{N}} & =\bigoplus_{C \in \mathbf{C}} g_{T}^{\widetilde{N}_{C}}, \quad h_{T}^{\widetilde{N}}=\bigoplus_{C \in \mathbf{C}} h_{T}^{\widetilde{N}_{C}}, \quad \Delta_{T}^{\widetilde{N}}=\bigoplus_{C \in \mathbf{C}} \Delta_{T}^{\widetilde{N}_{C}} .
\end{aligned}
$$

Recall that the function $\phi(T, y)$ was defined in Subsection 6.3. Since $\phi(T, y) \equiv T$ outside $B_{C, 2}$, it follows from (6.9) that there exists a closed cohomologous to $\omega$ 1form $\omega_{T}^{\prime} \in \Omega^{1}(\widetilde{M})$ whose critical set coincides with $\mathbf{C}$, and such that

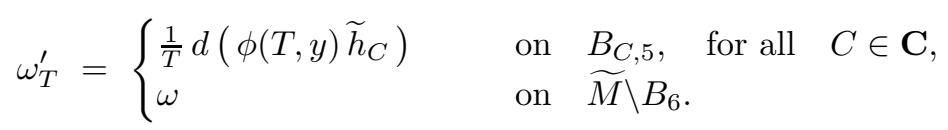


Consider the one-parameter family of connections

$$
\nabla_{T}^{\prime}=\nabla+T e\left(\omega_{T}^{\prime}\right) .
$$

By construction, the metrics $g_{T}^{\widetilde{N}}$ and $h_{T}^{\widetilde{N}}$ coincide on $B_{6} \backslash B_{3} \subset \widetilde{N}_{C} \cap M$, with $g_{1}^{\widetilde{N}}$ and $h_{1}^{\widetilde{N}}$, respectively. Hence, they can be extended to metrics $g_{T}^{\widetilde{M}}, h_{T}^{\widetilde{M}}$ on the whole $\widetilde{M}$ in such a way that the extensions do not depend on $T$ outside of $B_{3}$ and the restrictions of these metrics to the cylindrical part $\mathcal{T}$ have the form described in Subsection 4.1. For each $T \in \mathbb{R}$ we denote by $\nabla_{T}^{\prime *}$ the formal adjoint of $\nabla_{T}^{\prime}$ with respect to the metrics $g_{T}^{\widetilde{M}}$ and $h_{T}^{\widetilde{M}}$. Let

$$
\Delta_{T}^{\prime}=\frac{1}{T}\left(\nabla_{T}^{\prime} \nabla_{T}^{\prime *}+\nabla_{T}^{\prime *} \nabla_{T}^{\prime}\right)
$$

Let $\Delta_{T}^{\prime p}, \Delta_{T}^{\widetilde{N}, p}$ denote the restrictions of operators $\Delta_{T}^{\prime}, \Delta_{T}^{\widetilde{N}}$ to the space of $p$-forms.

7.2. Asymptotic of the spectrum of $\Delta_{T}^{\prime}$. Let $A$ be a self-adjoint operator with discrete spectrum. For any $\lambda>0$, we denote by $N(\lambda, A)$ the number of the eigenvalues of $A$ not exceeding $\lambda$ (counting multiplicity).

The following theorem plays a central role in our proof of Theorem 3.6.

Theorem 7.3. Let $\lambda_{p}(p=0,1, \ldots, \operatorname{dim} M)$ be the smallest non-zero eigenvalue of $\Delta_{1}^{\widetilde{N}, p}$. Then for any $\lambda_{p}>\varepsilon>0$ there exists $T_{\varepsilon}>0$ such that for all $T>T_{\varepsilon}$

$$
N\left(\lambda_{p}-\varepsilon, \Delta_{T}^{\prime p}\right)=\operatorname{dim} \operatorname{Ker} \Delta_{1}^{\widetilde{N}, p} .
$$

We will prove this theorem in the next section.

\subsection{Proof of Theorem 3.6. By Theorem 7.3,}

$$
N\left(\lambda_{p} / 2, \Delta_{T}^{\prime p}\right)=\operatorname{dim} \operatorname{Ker} \Delta_{1}^{\widetilde{N}, p}, \quad p=0,1, \ldots, \operatorname{dim} M .
$$

Let $\widetilde{\mathcal{F}}_{T}^{\prime}$ denote the flat vector bundle $\left(\widetilde{\mathcal{F}}, \nabla_{T}^{\prime}\right)$. Since $\omega$ is cohomologous to $\omega_{T}^{\prime}$, the cohomology groups $H^{p}\left(\widetilde{M}, \widetilde{U}_{c}^{-} ; \widetilde{\mathcal{F}}_{T}\right)$ and $H^{p}\left(\widetilde{M}, \widetilde{U}_{c}^{-} ; \widetilde{\mathcal{F}}_{T}^{\prime}\right)$ are isomorphic for all $T \in \mathbb{R}$.

Let us fix a generic $T>0$; cf. Subsection 1.9. Then the dimension of the cohomology $H^{p}\left(\widetilde{M}, \widetilde{U}_{c}^{-} ; \widetilde{\mathcal{F}}_{T}^{\prime}\right) \simeq H^{p}\left(\widetilde{M}, \widetilde{U}_{c}^{-} ; \widetilde{\mathcal{F}}_{T}\right)$ is equal to the generalized Novikov number $\beta_{i}(\xi, \mathcal{F})$; cf. Subsection 1.9. By Proposition [5.3, these numbers are equal to the dimension of the cohomology of the complex

$$
0 \rightarrow \widetilde{\Omega}_{T}^{0}(\widetilde{M}, \widetilde{\mathcal{F}}) \stackrel{\nabla_{T}^{\prime}}{\longrightarrow} \widetilde{\Omega}_{T}^{1}(\widetilde{M}, \widetilde{\mathcal{F}}) \stackrel{\nabla_{T}^{\prime}}{\longrightarrow} \cdots \stackrel{\nabla_{T}^{\prime}}{\longrightarrow} \widetilde{\Omega}_{T}^{n}(\widetilde{M}, \widetilde{\mathcal{F}}) \rightarrow 0 .
$$

Let $E_{T}^{p}(p=0,1, \ldots, \operatorname{dim} M)$ be the subspace of $\widetilde{\Omega}_{T}^{p}(\widetilde{M}, \widetilde{\mathcal{F}})$ spanned by the eigenvectors of $\Delta_{T}^{\prime p}$ corresponding to the eigenvalues $\lambda \leq \lambda_{p} / 2$. From (7.3) and Theorem 6.9 (2), we obtain

$$
\operatorname{dim} E_{T}^{p}=\operatorname{dim} H^{p-\operatorname{ind}(C)}\left(V_{C, \varepsilon}, \mathcal{F}_{\left.\right|_{V_{C, \varepsilon}}} \otimes o\left(\Sigma_{C}\right)_{\left.\right|_{V_{C, \varepsilon}}}\right) .
$$

Set

$$
\begin{aligned}
\mathcal{M}_{\varepsilon}(\lambda) & =\sum \lambda^{p} \operatorname{dim} E_{T}^{p} \\
& =\sum_{C \in \mathbf{C}} \lambda^{\operatorname{ind} C} \sum \lambda^{p} \operatorname{dim} H^{p-\operatorname{ind}(C)}\left(V_{C, \varepsilon}, \mathcal{F}_{\left.\right|_{V_{C, \varepsilon}}} \otimes o\left(\Sigma_{C}\right)_{\left.\right|_{V_{C, \varepsilon}}}\right) .
\end{aligned}
$$


Since the operator $\Delta_{T}^{\prime}$ commutes with $\nabla_{T}^{\prime}$, the pair $\left(E_{T}^{\bullet}, \nabla_{T}\right)$ is a subcomplex of (7.4), and the inclusion induces an isomorphism of cohomology

$$
H^{\bullet}\left(E_{T}^{\bullet}, \nabla_{T}^{\prime}\right) \cong H^{\bullet}\left(\widetilde{\Omega} \bullet(\widetilde{M}, \widetilde{\mathcal{F}}), \nabla_{T}^{\prime}\right) .
$$

Hence,

$$
\operatorname{dim} H^{p}\left(E_{T}^{\bullet}, \nabla_{T}^{\prime}\right)=\beta_{p}(\xi, \mathcal{F}), \quad p=0,1, \ldots, \operatorname{dim} M .
$$

The standard Morse theory arguments (cf., e.g., [6]) now imply that there exists a polynomial $\mathcal{Q}_{\varepsilon}(\lambda)$ with non-negative integer coefficients such that

$$
\mathcal{M}_{\varepsilon}(\lambda)-\mathcal{N}_{\omega, \mathcal{F}}(\lambda)=(1+\lambda) \mathcal{Q}_{\varepsilon}(\lambda) .
$$

Using the equality $\bigcap_{r>0} V_{C, \varepsilon}=C$ and the continuity of the Cech cohomology ([15. Ch. VIII §6.18]), we get

$$
\lim _{\varepsilon \rightarrow 0} \operatorname{dim} H^{p-\operatorname{ind}(C)}\left(V_{C, \varepsilon}, \mathcal{F}_{\left.\right|_{C, \varepsilon}} \otimes o\left(\Sigma_{C}\right)_{\left.\right|_{V_{C, \varepsilon}}}\right)=\operatorname{dim} \check{H}^{p-\operatorname{ind}(C)}\left(C, \mathcal{F}_{\left.\right|_{C}} \otimes o(C)\right) .
$$

Therefore, $\lim _{\varepsilon \rightarrow 0} \mathcal{M}_{\varepsilon}(\lambda)=\mathcal{M}_{\omega, \mathcal{F}}(\lambda)$. Letting $\varepsilon \rightarrow 0$ in (17.6) we obtain Theorem 3.6 .

\section{Comparison Between the Laplacians on the MANifold AND ON THE NORMAL BUNDLE}

In this section we prove Theorem 7.3. Our strategy will be to apply the IMS localization formula (cf. [14, 27]).

To simplify the notation we will omit the prime and will denote by $\Delta_{T}$ the operator defined in (7.2).

8.1. Estimate from above on $N\left(\lambda_{p}-\varepsilon, \Delta_{T}^{p}\right)$. We will first show that

$$
N\left(\lambda_{p}-\varepsilon, \Delta_{T}^{p}\right) \leq \operatorname{dim} \operatorname{Ker} \Delta_{1}^{\widetilde{N}, p}
$$

by estimating the operator $\Delta_{T}^{p}$ from below. We will use the technique of [27, adding some necessary modifications (see also [7]).

Recall that the notations $\tilde{N}, B_{r}, S_{r}$, etc. were defined in Subsection 7.1. Using Remark 1.6 we obtain an embedding $i: B_{r} \rightarrow \widetilde{M}, 0<r<6$.

Let $\tau: \widetilde{N} \rightarrow \mathbb{R}$ be a smooth function, such that $\tau(y) \leq 1$ for all $y \in B_{1}$ and

$$
\tau(x, t)=t \quad \text { for } \quad(x, t) \in S_{C, 1} \times[1, \infty) \subset \tilde{N} .
$$

Then $B_{r}=\tau^{-1}([0, r))$ for all $r>1$.

Let us fix a $C^{\infty}$ function $j:[0,+\infty) \rightarrow[0,1]$ such that $j(s)=1$ for $s \leq 4$, $j(s)=0$ for $s \geq 5$ and the function $\left(1-j^{2}\right)^{1 / 2}$ is $C^{\infty}$. We define functions $J, \bar{J} \in C^{\infty}(\tilde{N})$ by

$$
J(y)=j(\tau(y)) ; \quad \bar{J}(y)=\left(1-j(\tau(y))^{2}\right)^{\frac{1}{2}} .
$$

Using the diffeomorphism $i: B_{6} \rightarrow i\left(B_{6}\right)$ we can and we will consider $J, \bar{J}$ as functions on $\widetilde{M}$.

We identify the functions $J, \bar{J}$ with the corresponding multiplication operators. For operators $A, B$, we denote by $[A, B]=A B-B A$ their commutator.

The following version of IMS localization formula (cf. [14) is due to Shubin 27, Lemma 3.1]. 
Lemma 8.2. The following operator identity holds:

$$
\Delta_{T}^{p}=\bar{J} \Delta_{T}^{p} \bar{J}+J \Delta_{T}^{p} J+\frac{1}{2}\left[\bar{J},\left[\bar{J}, \Delta_{T}^{p}\right]\right]+\frac{1}{2}\left[J,\left[J, \Delta_{T}^{p}\right]\right] .
$$

Proof. Using the equality $J^{2}+\bar{J}^{2}=1$ we can write

$$
\Delta_{T}^{p}=J^{2} \Delta_{T}^{p}+\bar{J}^{2} \Delta_{T}^{p}=J \Delta_{T}^{p} J+\bar{J} \Delta_{T}^{p} \bar{J}+J\left[J, \Delta_{T}^{p}\right]+\bar{J}\left[\bar{J}, \Delta_{T}^{p}\right] .
$$

Similarly,

$$
\Delta_{T}^{p}=\Delta_{T}^{p} J^{2}+\Delta_{T}^{p} \bar{J}^{2}=J \Delta_{T}^{p} J+\bar{J} \Delta_{T}^{p} \bar{J}-\left[J, \Delta_{T}^{p}\right] J-\left[\bar{J}, \Delta_{T}^{p}\right] \bar{J} .
$$

Summing these identities and dividing by 2 we come to (8.2).

We will now estimate each of the summands on the right-hand side of (8.2).

Lemma 8.3. There exist $c>0, T_{0}>0$ such that, for any $T>T_{0}$,

$$
\bar{J} \Delta_{T}^{p} \bar{J} \geq c T \bar{J}^{2} I .
$$

Proof. Let $\eta$ be in $L^{2} \Omega^{p}\left((\widetilde{M}, \widetilde{\mathcal{F}}), g_{T}\right)$. Using (4.4) and Lemma 4.7 we can write

$$
\left\langle\bar{J} \Delta_{T}^{p} \bar{J} \eta, \eta\right\rangle=\frac{1}{T}\left\langle\Delta_{T}^{p} \bar{J} \eta, \eta\right\rangle+\langle a(x, t) \bar{J} \eta, \bar{J} \eta\rangle+T|\omega|^{2} \bar{J}^{2}\langle\eta, \eta\rangle .
$$

(Since the support of $\bar{J} \eta$ belongs to the set $M \backslash B_{4}$, where the scalar product does not depend on $T$, we can omit this index.) The first term of (8.4) is positive, the second is bounded, so the last term dominates and the estimate (8.3) holds.

Let $P_{T}^{p}: L^{2} \Omega^{p}(\widetilde{N}, \widetilde{\mathcal{F}}) \rightarrow \operatorname{Ker} \Delta_{T}^{\widetilde{N}, p}$ be the orthogonal projection. This is a finite rank operator on $L^{2} \Omega^{p}(\widetilde{N}, \widetilde{\mathcal{F}})$, and its rank equals $\operatorname{dim} \operatorname{Ker} \Delta_{T}^{\widetilde{N}, p}=\operatorname{dim} \operatorname{Ker} \Delta_{1}^{\widetilde{N}, p}$. Clearly,

$$
\Delta_{T}^{\widetilde{N}, p}+\lambda_{p} P_{T}^{p} \geq \lambda_{p} I .
$$

Using the identification $i: B_{6} \rightarrow i\left(B_{6}\right)$ we can consider $J P_{T}^{p} J$ and $J \Delta_{T}^{\widetilde{N}, p} J$ as operators on $\Omega^{p}(\widetilde{M}, \widetilde{\mathcal{F}})$.

By construction of the operators $\Delta_{T}$ and $\Delta_{T}^{\widetilde{N}}$ we have

$$
J \Delta_{T}^{p} J=J \Delta_{T}^{\widetilde{N}, p} J
$$

Hence, (8.5) implies the following.

Lemma 8.4. For any $T>0$

$$
J \Delta_{T}^{p} J+\lambda_{p} J P_{T}^{p} J \geq \lambda_{p} J^{2} I, \quad \operatorname{rk} J P_{T}^{p} J \leq \operatorname{dim} \operatorname{Ker} \Delta_{1}^{E, p} .
$$

For an operator $A: \Omega^{p}(\widetilde{M}, \widetilde{\mathcal{F}}) \rightarrow \Omega^{p}(\widetilde{M}, \widetilde{\mathcal{F}})$, we denote by $\|A\|_{T}$ its norm with respect to the $L^{2}$ scalar product on $\Omega^{p}(\widetilde{M}, \widetilde{\mathcal{F}})$ defined by metrics $g_{T}^{\widetilde{M}}$ and $h_{T}^{\widetilde{M}}$.

Lemma 8.5. There exists $C>0$ such that

$$
\|\left[J,\left[J, \Delta_{T}^{p}\right] \|_{T} \leq C T^{-1}, \quad T>0 .\right.
$$


Proof. The leading symbol of $\Delta_{T}^{p}$ is equal to the leading symbol of $\frac{1}{T} \Delta$ (here $\Delta=$ $\nabla \nabla^{*}+\nabla^{*} \nabla$ is the standard Laplacian). Hence (cf., for example, [4, Proposition 2.3])

$$
\|\left[J,\left[J, \Delta_{T}^{p}\right] \|_{T}=-\frac{2}{T}|d J|^{2} \leq-\frac{2}{T} \max _{y \in \bar{M}}|d J(y)| .\right.
$$

Here we can also omit the index $T$ of the norm of $d J$, since supp $d J \in B_{5} \backslash B_{4}$.

Similarly, one shows that

$$
\|\left[\bar{J},\left[\bar{J}, \Delta_{T}^{p}\right] \|_{T} \leq C T^{-1} .\right.
$$

From Lemma 8.2, Lemma 8.3, Lemma 8.4, Lemma 8.5 and (8.8) we get the following

Corollary 8.6. For any $\varepsilon>0$, there exists $T_{0}>0$ such that for any $T>T_{0}$

$$
\Delta_{T}^{p}+\lambda_{p} J P_{T}^{p} J \geq\left(\lambda_{p}-\varepsilon\right) I, \quad \operatorname{rk} J P_{T}^{p} J \leq \operatorname{dim} \operatorname{Ker} \Delta_{T}^{E, p} .
$$

The estimate (8.1) now follows from Corollary 8.6 and the following general lemma [26, p. 270].

Lemma 8.7. Assume that $A, B$ are self-adjoint operators in a Hilbert space $\mathcal{H}$ such that $\mathrm{rk} B \leq k$ and there exists $\mu>0$ such that

$$
\langle(A+B) u, u\rangle \geq \mu\langle u, u\rangle \quad \text { for any } \quad u \in \operatorname{Dom}(A) .
$$

Then $N(\mu-\varepsilon, A) \leq k$ for any $\varepsilon>0$.

8.8. Estimate from below on $N\left(\lambda_{p}-\varepsilon, \Delta_{T}^{p}\right)$. To prove Theorem 7.3 it remains to show that

$$
N\left(\lambda_{p}-\varepsilon, \Delta_{T}^{p}\right) \geq \operatorname{dim} \operatorname{Ker} \Delta_{T}^{\widetilde{N}, p} .
$$

Let $E_{T}^{p}$ be the subspace of $\Omega^{p}(\widetilde{M}, \widetilde{\mathcal{F}})$ spanned by the eigenvectors of $\Delta_{T}^{p}$ corresponding to the eigenvalues $\lambda \leq \lambda_{p}-\varepsilon$ and let $\Pi_{T}^{p}: \Omega^{p}(\widetilde{M}, \widetilde{\mathcal{F}}) \rightarrow E_{T}^{p}$ be the orthogonal projection. Then

$$
\operatorname{rk} \Pi_{T}^{p}=N\left(\lambda_{p}-\varepsilon, \Delta_{T}^{p}\right) .
$$

Using the diffeomorphism $i: B_{6} \rightarrow i\left(B_{6}\right)$ we can consider $J \Pi_{T}^{p} J$ as an operator on $L^{2} \Omega^{p}(E, \widetilde{\mathcal{F}})$. The proof of the following lemma does not differ from the proof of Corollary 8.6 .

Lemma 8.9. For any $\delta>\varepsilon$, there exists $T>0$ such that for any $t>T$

$$
\Delta_{T}^{E, p}+J \Pi_{T}^{p} J \geq\left(\lambda_{p}-\delta\right) I .
$$

The estimate (8.10) now follows from (8.11), Lemma 8.9 and Lemma 8.7

\section{ACKNOWLEDGMENT}

Some of the ideas used in this paper were developed several years ago in a joint unfinished project of M. Farber, M. Shubin and the first author. We are very grateful to M. Farber and M. Shubin for helping to develop these ideas and for allowing us to use them in this paper. 


\section{REFERENCES}

[1] S. Alesker and M. Braverman, Cohomology of a Hamiltonian T-space with involution, Preprint.

[2] M. F. Atiyah and R. Bott, The Yang-Mills equations over Riemann surfaces, Phil. Trans. R. Soc. London, ser A 308 (1983), 523-615. MR0702806 (85k:14006)

[3] _ The moment map and equivariant cohomology, Topology 23 (1984), 1-28. MR0721448 (85e:58041)

[4] N. Berline, E. Getzler, and M. Vergne, Heat kernels and Dirac operators, Springer-Verlag, 1992. MR1215720(94e:58130)

[5] J.-M. Bismut, The Witten complex and the degenerate Morse inequalities, J. Dif. Geom. 23 (1986), 207-240. MR0852155 (87m:58169)

[6] R. Bott, Morse theory indomitable, Publ. Math. IHES 68 (1988), 99-114. MR 1001450 (90f:58027)

[7] M. Braverman and M. Farber, The Novikov-Bott inequalities, C.R. Acad. Sci. Paris t. 321, Série I (1995), 897-902. MR1355849 (96i:58165)

[8] - Novikov inequalities with symmetry, C. R. Acad. Sci. Paris Sér. I Math. 323 (1996), 793-798. MR1416178(97h:57056)

[9] M. Braverman and M. Farber, Equivariant Novikov inequalities, K-Theory 12 (1997), 293318. MR $1485432(98 \mathrm{~m}: 57043)$

[10] _ Novikov type inequalities for differential forms with non-isolated zeros, Math. Proc. Cambridge Philos. Soc. 122 (1997), 357-375. MR1458239 (99b:58220)

[11] M. Braverman, O. Milatovich, and M. Shubin, Essential selfadjointness of Schrödingertype operators on manifolds, Russian Math. Surveys 57 (2002), 641-692. MR.1942115 (2004g:58021)

[12] P. Chernoff, Essential self-adjointness of powers of generators of hyperbolic equations, J. Functional Analysis 12 (1973), 401-414. MR0369890 (51:6119)

[13] C. Conley, Isolated invariant sets and the Morse index, CBMS Regional Conference Series in Mathematics, vol. 38, American Mathematical Society, Providence, R.I., 1978. MR0511133 (80c:58009)

[14] H.L. Cycon, R.G. Froese, W. Kirsch, and B. Simon, Schrödinger operators with applications to quantum mechanics and global geometry, Texts and Monographs in Physics, SpringerVerlag, 1987. MR:0883643 (88g:35003)

[15] A. Dold, Lectures on algebraic topology, Classics in Mathematics, Springer-Verlag, Berlin, 1995, Reprint of the 1972 edition. MR:1335915 (96c:55001)

[16] Y. Eliashberg and M. Gromov, Lagrangian intersection theory: finite-dimensional approach, Geometry of differential equations, Amer. Math. Soc. Transl. Ser. 2, vol. 186, Amer. Math. Soc., Providence, RI, 1998, pp. 27-118. MR1732407 (2002a:53102)

[17] M. Farber and E. Shustin, Witten deformation and polynomial differential forms, Geom. Dedicata 80 (2000), no. 1-3, 125-155. MR1762505 (2001i:58042)

[18] M.S. Farber, Sharpness of the Novikov inequalities, Functional Anal. Appl. 19 (1985), 49-59. MR0783706 (86g:58029)

[19] A. Floer, Witten's complex and infinite dimensional Morse theory, J. Diff. Geom 30 (1989), 207-221. MR1001276 (90d:58029)

[20] M. Gromov and B. Lawson, Positive scalar curvature and the Dirac operator on complete Riemannian manifolds, Inst. Hautes Études Sci. Publ. Math. (1983), no. 58, 295-408. MR0720933 (85g:58082)

[21] M. Hirsch, Differential topology, Graduate Texts in Math., vol. 33, Springer, Berlin, 1976. MR0448362(56:6669)

[22] F. C. Kirwan, Cohomology of quotients in symplectic and algebraic geometry, Mathematical Notes, vol. 31, Princeton University Press, Princeton, NJ, 1984. MR0766741 (86i:58050)

[23] S.P. Novikov, Multivalued functions and functionals. An analogue of the Morse theory, Soviet Math. Dokl. 260 (1981), 35-35. MR0630459 (83a:58025)

[24] The Hamiltonian formalism and a multivalued analogue of Morse theory, Russian Math. Surveys 37 (1982), 3-49, 248. MR0676612 (84h:58032)

[25] A. Pazhitnov, An analytic proof of the real part of Novikov's inequalities, Soviet Math. Dokl. 293 (1987), 1305-1307. MR0891557 (88j:57033) 
[26] M. Reed and B. Simon, Methods of modern mathematical physics IV: Analysis of operators, Academic Press, London, 1978. MR0493421 (58:12429c)

[27] M. A. Shubin, Semiclassical asymptotics on covering manifolds and Morse inequalities, Geom. Funct. Anal. 6 (1996), 370-409. MR1384616 (97i:58171)

[28] Spectral theory of the Schrödinger operators on non-compact manifolds: qualitative results, Spectral theory and geometry (Edinburgh, 1998), Cambridge Univ. Press, Cambridge, 1999, pp. 226-283. MR.1736869 (2001d:58037)

[29] C. Viterbo, Symplectic topology as the geometry of generating functions, Math. Ann. 292 (1992), no. 4, 685-710. MR:1157321 (93b:58058)

Department of Mathematics, Northeastern University, Boston, Massachusetts 02115

E-mail address: maxim@neu.edu

Department of Mathematics, Northeastern University, Boston, Massachusetts 02115

E-mail address: v.silantyev@neu.edu 